\title{
COEXISTENCE STATES AND GLOBAL ATTRACTIVITY FOR SOME CONVECTIVE DIFFUSIVE COMPETING SPECIES MODELS
}

\author{
JULIÁN LÓPEZ-GÓMEZ AND JOSÉ C. SABINA DE LIS
}

\begin{abstract}
In this paper we analyze the dynamics of a general competing species model with diffusion and convection. Regarding the interaction coefficients between the species as continuation parameters, we obtain an almost complete description of the structure and stability of the continuum of coexistence states. We show that any asymptotically stable coexistence state lies in a global curve of stable coexistence states and that Hopf bifurcations or secondary bifurcations only may occur from unstable coexistence states. We also characterize whether a semitrivial coexistence state or a coexistence state is a global attractor. The techniques developed in this work can be applied to obtain generic properties of general monotone dynamical systems.
\end{abstract}

\section{INTRODUCTION}

In this paper we analyze the existence, extinction, attractiveness, uniqueness and structure of the coexistence states (componentwise positive solution couples) of the following nonlinear boundary value problem

$$
\begin{gathered}
d_{1} \Delta u+\delta \nabla u-\ell_{1}(x) u=\lambda \ell_{2}(x) u-a(x) u^{2}-b(x) u v \\
-d_{2} \Delta v+\rho \nabla v-m_{1}(x) v=\mu m_{2}(x) v-c(x) u v-d(x) v^{2} \\
u=v=0 \text { on } \partial \Omega
\end{gathered}
$$

where $\Omega$ is a smooth bounded domain of $\mathbb{R}^{N}, N \geq 1$, of class $C^{2+\nu}$ for some $\nu \in(0,1)$, [15], the diffusivities $d_{1}$ and $d_{2}$ are positive real numbers, the convection coefficients $\delta$ and $\rho$ are vectors of $\mathbb{R}^{N}$ with constant entries and the coefficients $a(x), b(x), c(x), d(x), \ell_{i}(x), m_{i}(x), i=1,2$, belong to $C^{\nu}(\bar{\Omega})$; the space of Hölder continuous functions in $\bar{\Omega}$ with exponent $\nu$. We assume that $a(x), d(x), \ell_{2}(x)$ and $m_{2}(x)$ are positive in $\bar{\Omega}$ and that $b(x)$, $c(x)$ are nonnegative in $\Omega$. Throughout the paper we regard to $\lambda$ and $\mu$ as control parameters. Model (1.1) provides us with the steady-state solutions to the evolutionary problem

Received by the editors April 22, 1993 and, in revised form, November 15, 1993. 1991 Mathematics Subject Classification. Primary 35J55. 
$(1.2)$

$$
\begin{array}{cc}
\frac{\partial u}{\partial t}-d_{1} \Delta u+\delta \nabla u-\ell_{1}(x) u=\lambda \ell_{2}(x) u-a(x) u^{2}-b(x) u v & \text { in } \Omega \times[0, \infty), \\
\frac{\partial v}{\partial t}-d_{2} \Delta v+\rho \nabla v-m_{1}(x) v=\mu m_{2}(x) v-c(x) u v-d(x) v^{2} & (u(\cdot, 0), v(\cdot, 0))=\left(u_{0}, v_{0}\right), \text { in } \Omega .
\end{array}
$$

Under these assumptions, (1.2) is a model for competing species inhabiting the habitat $\Omega ; u(x), v(x)$ represent the population densities, $c(x), d(x)$ describe the limiting effects of crowding in each population, $d_{1}, d_{2}$ are the population diffusivities, $\delta, \rho$ take into account the external transport effects, and $b(x)$, $c(x)$ are the interaction coefficients between the species. The growth rates, $\ell_{1}(x)+\lambda \ell_{2}(x)$ and $m_{1}(x)+\mu m_{2}(x)$, are positive on favourable regions and not positive on unfavourable ones. We are assuming that the habitat is fully surrounded by inhospitable regions (homogeneous Dirichlet boundary conditions). Besides $(0,0)$, there are two types of component-wise nonnegative solution couples. Namely, the semitrivial coexistence states $(u, 0), u>0$, and $(0, v)$, $v>0$, and the coexistence states $(u, v), u, v>0$. Observe that if $w>0$ is a component of a solution couple of $(1.1)$ it follows from the maximum principle that $w(x)>0, x \in \Omega$, and $\frac{\partial w}{\partial n}(x)<0, x \in \partial \Omega$.

We now describe the results of this work and compare them with some previous work in the references. Given $d>0$, and $q, h \in C^{\nu}(\bar{\Omega})$ such that $h(x)>0, x \in \Omega, \sigma_{1}[d, q, h]$ will stand for the principal eigenvalue of $(-d \Delta+q) \varphi=\sigma h \varphi$ in $\Omega$ subject to homogeneous Dirichlet boundary conditions at the boundary. To simplify the notation we shall denote $q_{1}(x)=$ $\frac{|\delta|^{2}}{4 d_{1}}-\ell_{1}(x), q_{2}(x)=\frac{|\rho|^{2}}{4 d_{2}}-m_{1}(x), A(x)=a(x) e^{\frac{\delta . x}{2 d_{1}}}, B(x)=b(x) e^{\frac{\rho, x}{2 d_{2}}}, C(x)=$ $c(x) e^{\frac{\delta \cdot x}{2 d_{1}}}$ and $D(x)=d(x) e^{\frac{\rho \cdot x}{2 d_{2}}}$. The condition

$$
\lambda>\sigma_{1,1} \equiv \sigma_{1}\left[d_{1}, q_{1}, \ell_{2}\right], \quad \mu>\sigma_{1,2} \equiv \sigma_{1}\left[d_{2}, q_{2}, m_{2}\right],
$$

is necessary for the existence of a coexistence state of (1.1) (cf. Theorem 3.1) and so we shall impose it for the sequel. Then, (1.1) has two semitrivial coexistence states, denoted by $\left(\bar{\theta}_{\lambda}, 0\right)$ and $\left(0, \bar{\theta}_{\mu}\right)$, and it follows from Theorem 3.1 that

$$
\lambda>\sigma_{1}\left[d_{1}, q_{1}+B \bar{\theta}_{\mu}, \ell_{2}\right], \quad \mu>\sigma_{1}\left[d_{2}, q_{2}+C \bar{\theta}_{\lambda}, m_{2}\right],
$$

is sufficient for the existence of an asymptotically stable coexistence state. Condition (1.4) holds provided $\left\|b(x) e^{\frac{\rho . x}{2 d_{2}}}\right\|_{\infty}$ and $\left\|c(x) e^{\frac{\delta . x}{2 d_{1}}}\right\|_{\infty}$ are small enough. This can be accomplished either fixing $\delta, \rho$ and taking $b, c$ with $\|b(x)\|_{\infty}$ and $\|c(x)\|_{\infty}$ small, or fixing $b(x), c(x)$ and playing around with the geometry of $\Omega$ and the convection vectors $\delta, \rho$. This result is well known in the context of constant coefficients, [11]. Related results for some periodic-parabolic models can be found in [17] and [22]. All those results extend the existence theorem in [6]. In Theorem 3.4 we estimate the principal eigenvalues involved in (1.4) and find some explicit sufficient conditions for (1.4) in terms of the several coefficients in the setting of (1.1). Theorem 3.4 improves substantially some previous results of [4], [5], [12], and [14]. Moreover, as no variational characterization is used to get such estimates, Theorem 3.4 also holds for more general settings, e.g., periodic-parabolic systems. Theorem 4.1 shows that under 
condition (1.4) if (1.1) has a unique coexistence state then it is a global attractor for the solutions of (1.2) with positive initial data $u_{0}>0, v_{0}>0$ such that $u_{0}=v_{0}=0$ on $\partial \Omega$. This result is a substantial generalization of Theorem 4.1 of [6], which to the best of our knowledge is the only previous result. In Section 5 we first compute some fixed point indices by using the abstract theory developed in [1], [9] and [22] (cf. Theorem 5.1). Then, as a consequence of such theory we show that

$$
\lambda<\sigma_{1}\left[d_{1}, q_{1}+B \bar{\theta}_{\mu}, \ell_{2}\right], \quad \mu<\sigma_{1}\left[d_{2}, q_{2}+C \bar{\theta}_{\lambda}, m_{2}\right],
$$

is sufficient for the existence of a coexistence state with index -1 . So, generically unstable. The fixed point index also provides us with some multiplicity results. Namely, if the following relation is satisfied

$$
\left(\lambda-\sigma_{1}\left[d_{1}, q_{1}+B \bar{\theta}_{\mu}, \ell_{2}\right]\right) \cdot\left(\mu-\sigma_{1}\left[d_{2}, q_{2}+C \bar{\theta}_{\lambda}, m_{2}\right]\right)>0,
$$

then (1.1) has an odd number of coexistence states (generically). Observe that (1.6) holds if, and only if, (1.4) or (1.5) is satisfied. An analysis of bifurcation directions from the semitrivial coexistence states shows that (1.6) is not necessary for the existence of a coexistence state, [4], [11], [12]. In fact, from Theorem 5.1 we also obtain the following multiplicity result: If (1.1) has a coexistence state and the following condition is satisfied,

$$
\left(\lambda-\sigma_{1}\left[d_{1}, q_{1}+B \bar{\theta}_{\mu}, \ell_{2}\right]\right) \cdot\left(\mu-\sigma_{1}\left[d_{2}, q_{2}+C \bar{\theta}_{\lambda}, m_{2}\right]\right)<0,
$$

then (1.1) has an even number of coexistence states (generically). Moreover, the proof of Theorem 1 of [11] can be adapted to the current situation here to show that generically (1.1) has an asymptotically stable coexistence state if (1.7) is satisfied and (1.1) has some coexistence state. Furthermore, Theorem 4.4 shows that whenever (1.1) does not admit a coexistence state and (1.7) holds then some of the semitrivial coexistence states are global attractors. As the number of coexistence states of (1.1) may change with the size and the shape of $\Omega$, the previous results are optimal for general domains.

Now we pay attention to the problem of deciding whether equation (1.4), or (1.5), or (1.7), is satisfied or not. To solve this problem we analyze the sign of the functions $F(\beta)=\lambda-\sigma_{1}\left[d_{1}, q_{1}+\beta B \bar{\theta}_{\mu}, \ell_{2}\right], \beta \geq 0$, and $G(\gamma)=$ $\mu-\sigma_{1}\left[d_{2}, q_{2}+\gamma C \bar{\theta}_{\lambda}, m_{2}\right], \gamma \geq 0$. We obtain the following results. If $\{x \in$ $\Omega: b(x)=0\}$ has Lebesgue measure zero then there exists a unique $\beta_{s}>0$ such that $F(\beta)>0$ if $\beta<\beta_{s}, F\left(\beta_{s}\right)=0$, and $F(\beta)<0$ if $\beta>\beta_{s}$ (cf. Theorem 3.12 and Remark 3.13). If $\{x \in \Omega: b(x)=0\}$ has nonempty interior and $\lambda$ is large enough then $F(\beta)>0$ for all $\beta \geq 0$ (cf. Theorem 3.14). By symmetry, $G(\gamma)$ satisfies the same properties as $F(\beta)$. To see the biological meaning of these results, consider (1.1) with $b(x)$ replaced by $\beta b(x)$ and take $\mu$ large enough so that $\mu>\sigma_{1}\left[d_{2}, q_{2}+C \bar{\theta}_{\lambda}, m_{2}\right]$. Then (1.1) has a unique coexistence state if $\beta=0$, say $\left(u_{0}, v_{0}\right)$. Moreover, it follows from the Implicit Function Theorem that $(1.1)$ has a unique coexistence state if $\beta$ is small enough, say $(u(\beta), v(\beta))$, such that $(u(0), v(0))=\left(u_{0}, v_{0}\right)$. It follows from Theorem 4.1 that $(u(\beta), v(\beta))$ is a global attractor. Therefore, if the interactions between the species are small enough nor species can drive the 
other to extinction. Assume in addition that $\{x \in \Omega: b(x)=0\}$ has Lebesgue measure zero. Then it follows from Theorem 3.12 that (1.1) does not admit a coexistence state if $\beta$ is large enough and hence it follows from Theorem 4.4 that $\left(0, \bar{\theta}_{\mu}\right)$ is a global attractor for the positive solutions of (1.2). Therefore, if the interaction of $v$ on $u$ is large enough, then $v$ drives $u$ to extinction. Now assume that $\{x \in \Omega: b(x)=0\}$ has nonempty interior. Then $u$ is free of $v$ in some subdomain of the inhabiting region and if the birthrate of $u$ is large enough then $v$ does not drive necessarily to $u$ to extinction, because (1.4) holds and so (1.1) has an asymptotically coexistence state.

In Section 8, assuming that $\mu>\sigma_{1}\left[d_{2}, q_{2}+C \bar{\theta}_{\lambda}, m_{2}\right]$ and that $b(x)>0$, $c(x)>0, x \in \Omega$, we analyze the global behaviour of the branch of coexistence states $(u(\beta), v(\beta))$ constructed above. Such a branch satisfies the following. Either (i) it is a regular curve which can be continued up to degenerate at the semitrivial solution $\left(0, \bar{\theta}_{\mu}\right)$ at $\beta=\beta_{s}$, or (ii) it is a regular curve which can be continued up to reach a subcritical turning point, which still is a regular point of the curve. In case (i) the solution $(u(\beta), v(\beta))$ is asymptotically stable for all $\beta \in\left[0, \beta_{s}\right)$, except at most for a finite number of $\beta$ (hysteresis points). In case (ii) there exists $\beta_{t}>0$ such that $(u(\beta), v(\beta))$ is asymptotically stable for all $\beta \in\left[0, \beta_{t}\right)$, except at most for a finite number of $\beta$. Then, it turns backward becoming unstable. Moreover, if $\beta_{t}<\beta_{s}$ then there is some range of $\beta$ for which (1.1) has at least three stable coexistence states (cf. Theorem 8.1). In fact, any asymptotically stable coexistence state lies in a global curve of coexistence states. Global in the sense that it can be continued up to degenerate at a semitrivial coexistence state or up to reach a regular turning point, at which the stability gets lost (cf Remark 8.2 and Theorem 8.3). In particular, when $\beta$ varies no Hopf bifurcation nor secondary bifurcation can occur from any stable coexistence state. To the best of our knowledge no previous result of this nature is available in the literature. Our results complement the analysis carried out in [3] concerning the topological nature of the continuum of coexistence states of (1.1) in the case of constant coefficients. The technical tools used to show these results are given in Sections 6 and 7. As the results in such sections are of interest by themselves we wrote them separately. In Section 6 we use the framework introduced in [23] to obtain a general maximum principle for quasimonotone systems. In Section 7 we use the maximum principle to characterize the stability of the coexistence states of (1.1). Some related work for the case of one equation can be found in [1].

Finally, in Section 9 we bring together all the results above to characterize the supremum of the set of $\hat{\beta}$ such that (1.1) has a unique coexistence state for all $\beta \in[0, \hat{\beta})$. Such characterization is carried out by means of the spectral radius of a composition of four compact strongly positive endomorphisms; two resolvents of elliptic operators and two multiplication operators. Let $\beta_{g a}$ denote this supremum. Either $\beta_{g a}=\beta_{s}$ and then (1.1) has a unique coexistence state for all $\beta \in\left[0, \beta_{s}\right)$, which is a global attractor and degenerates at $\left(0, \bar{\theta}_{\mu}\right)$, or $\beta_{g a}<\beta_{s}$. If $\beta_{g a}<\beta_{s}$ then (1.1) has two coexistence states at $\beta_{g a}$ (at least) and there exists $\varepsilon>0$ such that either (1.1) has three coexistence states for $\beta \in\left(\beta_{g a}, \beta_{g a}+\varepsilon\right)$, or (1.1) has a unique coexistence state for $\beta \in\left(\beta_{g a}, \beta_{g a}+\varepsilon\right)$. We also find some lower estimates for $\beta_{g a}$ in terms of the several parameters and coefficients involved in the setting of (1.1). 


\section{Preliminaries and notations. The logistic equation}

For fixed $\nu>0$, we consider the Banach spaces $U \equiv\left\{w \in C^{2+\nu}(\overline{\boldsymbol{\Omega}})\right.$ : $\left.\left.w\right|_{\partial \Omega} \equiv 0\right\}$ and $V \equiv C^{\nu}(\bar{\Omega})$ ordered by the cones of nonnegative functions $P_{U} \equiv\{w \in U: w \geq 0\}$ and $P_{V} \equiv\{w \in V: w \geq 0\}$, respectively. The interior of a cone $P$ will be denoted by $\dot{P}$ and we shall write $f \geq g$ if $f-g \in P, f>g$ if $f-g \in P-\{0\}$ and $f \gg g$ if $f-g \in \dot{P}$. The interior of $P_{U}$ is the set of functions $w \in U$ such that $w(x)>0$ for all $x \in \Omega$ and $\frac{\partial w}{\partial n}(x)<0$ for all $x \in \partial \Omega$, where $n$ is the outward unit normal to $\Omega$ at $\partial \Omega$.

By setting $u=e^{\frac{\delta x}{2 d_{1}}} u_{1}, v=e^{\frac{\rho \cdot x}{2 d_{2}}} v_{1}$ and renaming $u_{1}$ and $v_{1}$ as $u$ and $v$, respectively, the parabolic problem (1.2) becomes

$$
\begin{gathered}
\frac{\partial u}{\partial t}-d_{1} \Delta u+\left(\frac{|\delta|^{2}}{4 d_{1}}-\ell_{1}\right) u=\lambda \ell_{2}(x) u-a(x) e^{\frac{\delta . x}{2 d_{1}}} u^{2}-b(x) e^{\frac{\rho \cdot x}{2 d_{2}}} u v, \\
\frac{\partial v}{\partial t}-d_{2} \Delta v+\left(\frac{|\rho|^{2}}{4 d_{2}}-m_{1}\right) v=\mu m_{2}(x) v-c(x) e^{\frac{\delta . x}{2 d_{1}}} u v-d(x) e^{\frac{\rho . x}{2 d_{2}}} v^{2}, \\
u(\cdot, t), v(\cdot, t) \in U, t>0, \quad(u(x, 0), v(x, 0))=\left(u_{0}(x), v_{0}(x)\right), x \in \Omega .
\end{gathered}
$$

In particular, this change of variables transforms (1.1) into

$$
\begin{aligned}
& -d_{1} \Delta u+q_{1}(x) u=\lambda \ell_{2}(x) u-A(x) u^{2}-B(x) u v \\
& -d_{2} \Delta v+q_{2}(x) v=\mu m_{2}(x) v-C(x) u v-D(x) v^{2}
\end{aligned} \quad(u, v \in U),
$$

where

$$
\begin{gathered}
q_{1}(x)=\frac{|\delta|^{2}}{4 d_{1}}, \quad q_{2}(x)=\frac{|\rho|^{2}}{4 d_{2}}, \quad A(x)=a(x) e^{\frac{\delta . x}{2 d_{1}}}, \\
B(x)=b(x) e^{\frac{\rho \cdot x}{2 d_{2}}}, \quad C(x)=c(x) e^{\frac{\delta x x}{2 d_{1}}}, \quad D(x)=d(x) e^{\frac{\rho \cdot x}{2 d_{2}}} .
\end{gathered}
$$

In absence of the other, each species satisfies a logistic boundary value problem of the form

$$
-d \Delta w+q(x) w=\gamma h(x) w-E(x) w^{2}, \quad w \in U,
$$

where $q, h, E \in C^{\nu}(\bar{\Omega}), E(x)>0$ for every $x \in \bar{\Omega}, h>0$, and $d, \gamma \in \mathbb{R}$, $d>0$. It follows from the strong maximum principle that any nontrivial positive solution of (2.4) lies in $\dot{P}_{U}$. A well-known result in [7] shows that the linear weighted boundary value problem

$$
-d \Delta \varphi+q(x) \varphi=\sigma h(x) \varphi, \quad \varphi \in U,
$$

has a unique principal eigenvalue. Hereafter we shall denote it by $\sigma_{1}[d, q, h]$. By principal eigenvalue we mean a value of $\sigma$ (not necessarily positive) associated with which there is a positive eigenfunction; $\varphi_{1}[d, q, h]$ will stand for the corresponding eigenfunction, unique up to multiplicative constants. The principal eigenfunction satisfies $\varphi_{1}[d, q, h] \gg 0$. The following result collects some well-known properties of $\sigma_{1}[d, q, h]$ (cf. [7], [17] and references therein).

Lemma 2.1. (i) If $q_{1}<q_{2}$ then $\sigma_{1}\left[d, q_{1}, h\right]<\sigma_{1}\left[d, q_{2}, h\right]$.

(ii) If $0<h_{1}<h_{2}$ then $\sigma_{1}\left[d, q, h_{1}\right]>\sigma_{1}\left[d, q, h_{2}\right]$.

(iii) The following variational characterization holds:

$$
\sigma_{1}[d, q, h]=\inf _{u \in H_{0}^{1}(\Omega)-\{0\}}\left\{\frac{\int_{\Omega}\left[d|\nabla u|^{2}+q(x) u^{2}\right] d x}{\int_{\Omega} h(x) u^{2} d x}\right\} .
$$


The main features concerning existence, uniqueness and attractivity of coexistence states of (2.4) are collected in the following result, [17].

Theorem 2.2. The logistic problem (2.4) admits a coexistence state if, and only if, $\gamma>\sigma_{1}[d, q, h]$. If this is the case, the coexistence state is unique and globally asymptotically stable (with respect to positive initial conditions $w_{0} \in P_{V}-\{0\}$ ). If $\gamma \leq \sigma_{1}[d, q, h]$ the trivial solution $w \equiv 0$ is globally asymptotically stable (with respect to initial conditions $w_{0} \in P_{V}$ ).

Throughout this work $\theta_{\gamma}[d, q, h, E]$ will stand for the maximal nonnegative solution of equation (2.4), i.e., $\theta_{\gamma}[d, q, h, E] \gg 0$ if $\gamma>\sigma_{1}[d, q, h]$ and $\theta_{\gamma}[d, q, h, E]=0$ if $\gamma \leq \sigma_{1}[d, q, h]$. For later use, we have to introduce some properties of the coexistence branch $\theta_{\gamma}[d, q, h], \gamma>\sigma_{1}[d, q, h]$. We collect them in the following result.

Theorem 2.3. (a) The mapping $\gamma \rightarrow \theta_{\gamma}[d, q, h, E], \gamma>\sigma_{1}[d, q, h]$, is analytic. Moreover,

$$
\left.\frac{d \theta_{\gamma}[d, g, h, E]}{d \gamma}\right|_{\gamma=\sigma_{1}[d, q, h]}=\frac{1}{\gamma_{1}} \varphi_{1}[d, q, h],
$$

where $\gamma_{1}=\frac{\int_{\Omega} E(x) \varphi_{1}^{3} d x}{\int_{\Omega} h(x) \varphi_{1}^{2} d x}$ and $\varphi_{1} \equiv \varphi_{1}[d, q, h]$.

(b) The mappings $\gamma \rightarrow \theta_{\gamma}[d, q, h, E]$ and $h \rightarrow \theta_{\gamma}[d, q, h, E]$ are increasing and the mapping $q \rightarrow \theta_{\gamma}[d, q, h, E]$ is decreasing. In any case the remaining arguments are kept fixed.

(c) There exists a positive constant

$$
M=M(d, q, h, E, \Omega) \geq \frac{\left\|\varphi_{1}\right\|_{\infty, \Omega}}{\gamma_{1}}
$$

such that $\left\|\theta_{\gamma}[d, q, h, E]\right\|_{\infty, \Omega} \leq M\left(\gamma-\sigma_{1}[d, q, h]\right)$ if $\gamma>\sigma_{1}[d, q, h]$.

Proof. (a) The analyticity follows because the branch $(\gamma, w)=\left(\gamma, \theta_{\gamma}[d, q, h]\right)$ may be obtained by global analytic continuation from $(\gamma, w)=\left(\sigma_{1}[d, q, h], 0\right)$ using the main theorem in [8] and the fact that any positive solution of (2.4) is nondegenerate. Moreover, if $\gamma \simeq \sigma_{1}[d, q, h]$ the branch $\left(\gamma, \theta_{\gamma}[d, q, h]\right)$ may be parametrized by an auxiliary parameter $s$ in the form $w=s \varphi_{1}+$ $\sum_{j=1}^{\infty} u_{j} s^{j+1}, \gamma=\sigma_{1}+\sum_{j=1}^{\infty} \gamma_{j} s^{j}$, [8]. By substituting these expressions into (2.4) and identifying the $s$-terms in both sides it follows that

$$
\left(-d \Delta+q(x)-\sigma_{1}[d, q, h] h(x)\right) u_{1}=\gamma_{1} h \varphi_{1}-E(x) \varphi_{1}^{2}, \quad u_{1} \in U .
$$

Finally Fredholm's alternative provides us with the value of $\gamma_{1}$.

(b) Assume $\lambda<\mu$. Then the function $\bar{u}=\theta_{\mu}[d, q, h, E]$ satisfies $-d \Delta \bar{u}+$ $q \bar{u} \geq \lambda h(x) \bar{u}-E(x) \bar{u}^{2}$ and the maximum principle implies $\theta_{\lambda} \leq \theta_{\mu}$. In fact, $\theta_{\mu} \gg \theta_{\lambda}$ if $\mu>\sigma_{1}$. Arguing as above the rest of (b) follows.

(c) Observe that $\frac{\left\|\theta_{\gamma}\right\|_{\infty, \Omega}}{\gamma-\sigma_{1}}$ is bounded in $\left[\sigma_{1}, \sigma_{1}+\delta\right]$ for every $\delta>0$. Thus, it is enough to show that $\frac{\left\|\theta_{\gamma}\right\|_{\infty, \Omega}}{\gamma-\sigma_{1}}$ is bounded for $\gamma \geq \sigma_{1}+\delta$. From part (b) we find that $\theta_{\gamma}[d, q, h, E] \leq \theta_{\gamma}\left[d, q,\|h\|_{\infty, \Omega}, \min _{\bar{\Omega}} E\right]$. Let $x_{0} \in \Omega$ be the point at which $\theta_{\gamma}\left[d, q,\|h\|_{\infty, \Omega}, \min _{\bar{\Omega}} E\right]$ attains its maximum. Then

$$
-d \Delta \theta_{\gamma}\left[d, q,\|h\|_{\infty, \Omega}, \min _{\bar{\Omega}} E\right]\left(x_{0}\right) \geq 0
$$


and hence

$$
\theta_{\gamma}\left[d, q,\|h\|_{\infty, \Omega}, \min _{\bar{\Omega}} E\right]\left(x_{0}\right) \leq \frac{\gamma\|h\|_{\infty, \Omega}-q\left(x_{0}\right)}{\min _{\bar{\Omega}} E} \leq \frac{\gamma\|h\|_{\infty, \Omega}-\hat{q}}{\min _{\bar{\Omega}} E},
$$

where $\hat{q}=\min _{\bar{\Omega}} q$. Therefore,

$$
\frac{\left\|\theta_{\gamma}[d, q, h, E]\right\|_{\infty, \Omega}}{\gamma-\sigma_{1}[d, q, h]} \leq \frac{\|h\|_{\infty, \Omega}}{\min _{\bar{\Omega}} E} \cdot \frac{\gamma-\hat{q}\|h\|_{\infty, \Omega}^{-1}}{\gamma-\sigma_{1}[d, q, h]} .
$$

The second factor on the right-hand side of this relation goes to unity as $\gamma \rightarrow \infty$. This completes the proof. The lower bound (2.5) follows readily from part (a).

\section{EXISTENCE OF STABLE COEXISTENCE STATES. EXTINCTION}

3.1 An existence result. Hereafter we shall denote

$$
\begin{gathered}
\bar{\theta}_{\lambda} \equiv \theta_{\lambda}\left[d_{1}, q_{1}, \ell_{2}, A\right], \quad \bar{\theta}_{\mu} \equiv \theta_{\mu}\left[d_{2}, q_{2}, m_{2}, D\right], \\
\underline{\theta}_{\lambda} \equiv \theta_{\lambda}\left[d_{1}, q_{1}+B \bar{\theta}_{\mu}, \ell_{2}, A\right], \quad \underline{\theta}_{\mu} \equiv \theta_{\mu}\left[d_{2}, q_{2}+C \bar{\theta}_{\lambda}, m_{2}, D\right],
\end{gathered}
$$

where $q_{1}, q_{2}, A, B, C$ and $D$ are defined in (2.3). The first result concerning the existence and stability of coexistence states of (2.2) is the following.

Theorem 3.1. The following assertions are true:

(a) Any coexistence state $(u, v)$ of (2.2) satisfies the estimates

$$
\underline{\theta}_{\lambda} \leq u \leq \bar{\theta}_{\lambda}, \quad \underline{\theta}_{\mu} \leq v \leq \bar{\theta}_{\mu} .
$$

(b) If either $\lambda \leq \sigma_{1}\left[d_{1}, q_{1}, \ell_{2}\right]$ or $\mu \leq \sigma_{1}\left[d_{2}, q_{2}, m_{2}\right]$ then (2.2) does not admit a coexistence state.

(c) Assume

$$
\lambda>\sigma_{1}\left[d_{1}, q_{1}+B \bar{\theta}_{\mu}, \ell_{2}\right], \quad \mu>\sigma_{1}\left[d_{2}, q_{2}+C \bar{\theta}_{\lambda}, m_{2}\right] .
$$

Then (2.2) has a coexistence state which is asymptotically stable.

Proof. Let $(u, v)$ be a componentwise nonnegative solution couple of (2.2). Then $-d_{1} \Delta u+q_{1} u \leq \lambda \ell_{2} u-A u^{2}$, and hence $u(x)$ is a nonnegative lower solution of

$$
-d_{1} \Delta z+q_{1} z=\lambda \ell_{2} z-A z^{2}, \quad z \in U .
$$

As $\bar{\theta}_{\lambda}$ is the maximal nonnegative solution of (3.3), a comparison involving the maximum principle shows that $0 \leq u \leq \bar{\theta}_{\lambda}$. Similarly, $0 \leq v \leq \bar{\theta}_{\mu}$. These estimates show part (b). Now, substituting $v \leq \bar{\theta}_{\mu}$ (resp. $u \leq \bar{\theta}_{\lambda}$ ) into the first equation (resp. the second one) of (2.2) and arguing as before (3.1) follows.

We now show part (c). It follows from (3.2) that $\underline{\theta}_{\lambda} \gg 0$ and that $\underline{\theta}_{\mu} \gg 0$. Set $\underline{u}_{0}:=\underline{\theta}_{\lambda}, \bar{u}_{0}:=\bar{\theta}_{\lambda}, \underline{v}_{0}:=\underline{\theta}_{\mu}$ and $\bar{v}_{0}:=\bar{\theta}_{\mu}$. Lemma $2.1(\mathrm{i})$ implies $\underline{u}_{0} \ll \bar{u}_{0}$ and $\underline{v}_{0} \ll \bar{v}_{0}$. Moreover, the couples $\left(\underline{u}_{0}, \bar{v}_{0}\right)$ and $\left(\bar{u}_{0}, \underline{v}_{0}\right)$ satisfy

$$
\begin{aligned}
& -d_{1} \Delta \underline{u}_{0}+q_{1}(x) \underline{u}_{0} \leq \lambda \ell_{2}(x) \underline{u}_{0}-A(x) \underline{u}_{0}^{2}-B(x) \underline{u}_{0} \bar{v}_{0}, \\
& -d_{2} \Delta \bar{v}_{0}+q_{2}(x) \bar{v}_{0} \geq \mu m_{2}(x) \bar{v}_{0}-C(x) \underline{u}_{0} \bar{v}_{0}-D(x) \bar{v}_{0}^{2}, \\
& -d_{1} \Delta \bar{u}_{0}+q_{1}(x) \bar{u}_{0} \geq \lambda \ell_{2}(x) \bar{u}_{0}-A(x) \bar{u}_{0}^{2}-B(x) \bar{u}_{0} \underline{v}_{0}, \\
& -d_{2} \Delta \underline{v}_{0}+q_{2}(x) \underline{v}_{0} \leq \mu m_{2}(x) \underline{v}_{0}-C(x) \bar{u}_{0} \underline{v}_{0}-D(x) \underline{v}_{0}^{2} .
\end{aligned}
$$


Thus, it follows from the results in [25] and [26] (cf. also [21], [30]) that (2.2) has a coexistence state. In fact, (2.2) has a coexistence state which is asymptotically stable, [11], [17].

For the sake of completeness we give here a simple algorithm which converges to a coexistence state of (2.2). Consider the sequences $\left(\underline{u}_{n}, \bar{v}_{n}\right),\left(\bar{u}_{n}, \underline{v}_{n}\right)$, $n \geq 0$, defined from $\left(\underline{u}_{0}, \bar{v}_{0}\right)$ and $\left(\bar{u}_{0}, \underline{v}_{0}\right)$ as follows. The couple $\left(\underline{u}_{n+1}, \bar{v}_{n+1}\right)$ is the unique componentwise positive solution $(u, v) \in U^{2}$ of

$$
\begin{gathered}
-d_{1} \Delta u+q_{1}(x) u=\lambda \ell_{2}(x) u-A(x) u^{2}-B(x) \bar{v}_{n} u, \\
-d_{2} \Delta v+q_{2}(x) v=\mu m_{2}(x) v-D(x) v^{2}-C(x) \underline{u}_{n} v .
\end{gathered}
$$

Similarly, $\left(\bar{u}_{n+1}, \underline{v}_{n+1}\right)$ is defined as the unique componentwise positive solution $(u, v) \in U^{2}$ of

$$
\begin{gathered}
-d_{1} \Delta u+q_{1}(x) u=\lambda \ell_{2}(x) u-A(x) u^{2}-B(x) \underline{v}_{n} u, \\
-d_{2} \Delta v+q_{2}(x) v=\mu m_{2}(x) v-D(x) v^{2}-C(x) \bar{u}_{n} v .
\end{gathered}
$$

The existence of such solutions follows from [29]. The uniqueness follows from Theorem 2.2. Moreover, it follows from the maximum principle that $\underline{u}_{n}$ and $\underline{v}_{n}$ are increasing while $\bar{u}_{n}$ and $\bar{v}_{n}$ are decreasing, [27]. Furthermore, $\underline{u}_{0} \leq$ $\underline{u}_{n} \leq \bar{u}_{0}$ and $\underline{v}_{0} \leq \underline{v}_{n} \leq \bar{v}_{0}$. Therefore, the limits $\left(u_{*}, v^{*}\right)=\lim _{n \rightarrow \infty}\left(\underline{u}_{n}, \bar{v}_{n}\right)$, $\left(u^{*}, v_{*}\right)=\lim _{n \rightarrow \infty}\left(\bar{u}_{n}, \underline{v}_{n}\right)$, hold uniformly in $\bar{\Omega}$ and provide us with classical solutions of (2.2), [29]. A priori, we do not know whether or not $\left(u^{*}, v_{*}\right)=$ $\left(u_{*}, v^{*}\right)$. These features show that $(2.2)$ has at least one asymptotically stable coexistence state.

Remark 3.2. If there exist lower and upper solutions such that $0 \ll \underline{u}_{0} \leq u \leq \bar{u}_{0}$ and $0 \ll \underline{v}_{0} \leq v \leq \bar{v}_{0}$, then (2.2) has a coexistence state which is asymptotically stable. Condition (3.2) guarantees the existence of such lower and upper solutions.

Remark 3.3. The set of values of $(\lambda, \mu)$ satisfying (3.2) is an open set of the $(\lambda, \mu)$-plane in between the curves

$$
C_{(0, v)} \equiv \lambda=\sigma_{1}\left[d_{1}, q_{1}+B \bar{\theta}_{\mu}, \ell_{2}\right], \quad C_{(u, 0)} \equiv \mu=\sigma_{1}\left[d_{2}, q_{2}+C \bar{\theta}_{\lambda}, m_{2}\right] .
$$

Both curves pass through by the point $(\lambda, \mu)=\left(\sigma_{1}\left[d_{1}, q_{1}, \ell_{2}\right], \sigma_{1}\left[d_{2}, q_{2}, m_{2}\right]\right)$. The fact that the set of values of $(\lambda, \mu)$ satisfying (3.2) is empty or not depends on the relative location of the curves $C_{(u, 0)}$ and $C_{(0, v)}$. Theorem 3.4 below will provide us with some global wedge-type regions in the $(\lambda, \mu)$-plane with vertex at $\left(\sigma_{1}\left[d_{1}, q_{1}, \ell_{2}\right], \sigma_{1}\left[d_{2}, q_{2}, m_{2}\right]\right)$ and satisfying (3.2). We have denoted by $C_{(u, 0)}$ the curve $\mu=\sigma_{1}\left[d_{2}, q_{2}+C \bar{\theta}_{\lambda}, m_{2}\right]$ because given $\lambda>\sigma_{1}\left[d_{1}, q_{1}, \ell_{2}\right]$ if we regard $\mu$ as a bifurcation parameter then the value $\mu=\sigma_{1}\left[d_{2}, q_{2}+C \bar{\theta}_{\lambda}, m_{2}\right]$ is a bifurcation point to coexistence states from the branch of semitrivial coexistence states $(u, 0)=\left(\bar{\theta}_{\lambda}, 0\right)$, [2], [12]. For the same reason we have denoted the other curve by $C_{(0, v)}$.

We now compare our result with the existence theorem found in [6]. In [6] the following simplified model was analyzed:

$$
-\Delta u=\lambda u-a u^{2}-b u v, \quad-\Delta v=\mu v-c u v-d v^{2}, \quad u, v \in U,
$$


where $a, b, c, d$ are positive real numbers. Set $\theta_{\lambda}[a]=\theta_{\lambda}[1,0,1, a], \sigma_{1}[q]=$ $\sigma_{1}[1, q, 1], \varphi_{1}[q]=\varphi_{1}[1, q, 1]$ and $\theta_{\lambda}=\theta_{\lambda}[1]$. Then $\theta_{\lambda}[a]=\frac{\theta_{\lambda}}{a}$ and it follows from Krein-Rutman's Theorem that $\sigma_{1}\left[\theta_{\lambda}\right]=\lambda$ and $\varphi_{1}\left[\theta_{\lambda}\right]=\theta_{\lambda}$. Thus, condition (3.2) becomes

$$
\lambda>\sigma_{1}\left[b \theta_{\mu}[d]\right]=\sigma_{1}\left[\frac{b}{d} \theta_{\mu}\right], \quad \mu>\sigma_{1}\left[c \theta_{\lambda}[a]\right]=\sigma_{1}\left[\frac{c}{a} \theta_{\lambda}\right] .
$$

Denote $g(\lambda)=\sigma_{1}\left[c \theta_{\lambda}[a]\right]$ and $h(\mu)=\sigma_{1}\left[b \theta_{\mu}[d]\right]$. Then $g=g(\lambda)$ and $h=$ $h(\mu)$ are smooth strictly increasing functions if $\lambda>\sigma_{1}[0]$ and $\mu>\sigma_{1}[0]$, respectively, such that $g\left(\sigma_{1}[0]\right)=h\left(\sigma_{1}[0]\right)=\sigma_{1}[0]$. A pair $(\lambda, \mu)$ satisfies (3.5) if, and only if,

$$
h(g(\lambda))<\lambda, \quad g(\lambda)<\mu<h^{-1}(\lambda) .
$$

Moreover, when $h(g(\lambda)) \geq \lambda$ no $\mu$ satisfies $g(\lambda)<\mu<h^{-1}(\lambda)$. Assume

$$
b<d, \quad c<a .
$$

Then $h(g(\lambda))=\sigma_{1}\left[\frac{b}{d} \theta_{g(\lambda)}\right]<\sigma_{1}\left[\theta_{g(\lambda)}\right]=g(\lambda)=\sigma_{1}\left[\frac{c}{a} \theta_{\lambda}\right]<\sigma_{1}\left[\theta_{\lambda}\right]=\lambda$ and hence $h(g(\lambda))<\lambda$ for all $\lambda>\sigma_{1}$. Thus, whenever (3.7) is satisfied the set of values of $(\lambda, \mu)$ satisfying (3.6) is an unbounded wedge-type region with vertex at $\left(\sigma_{1}[0], \sigma_{1}[0]\right)$. As $\sigma_{1}\left[\frac{c}{a} \theta_{\lambda}\right]<\sigma_{1}\left[\frac{c}{a} \lambda\right]=\sigma_{1}[0]+\frac{c}{a} \lambda$ and $\sigma_{1}\left[\frac{b}{d} \theta_{\mu}\right]<\sigma_{1}\left[\frac{b}{d} \mu\right]=$ $\sigma_{1}[0]+\frac{b}{d} \mu$ for $\lambda>\sigma_{1}[0]$ and $\mu>\sigma_{1}[0]$, the condition

$$
\lambda>\sigma_{1}[0]+\frac{b}{d} \mu, \quad \mu>\sigma_{1}[0]+\frac{c}{a} \lambda
$$

is sufficient for (3.5) (or (3.6)). The set of values of $(\lambda, \mu)$ satisfying (3.8) is nonempty if, and only if, $\frac{c}{a} \frac{b}{d}<1$. Moreover, if $\frac{c}{a} \frac{b}{d}<1$ then (3.8) is a wedge with edges $\lambda=\sigma_{1}[0]+\frac{b}{d} \mu, \mu=\sigma_{1}[0]+\frac{c}{a} \lambda$ and vertex at $\left(\frac{a(b+d)}{a d-b c} \sigma_{1}[0]\right.$, $\left.\frac{d(a+c)}{a d-b c} \sigma_{1}[0]\right)$. In particular, Theorem 3.1 is sharper than Theorem 4.1 of [6], which shows that (3.8) is sufficient for the existence of a coexistence state. It is even possible to show that condition (3.5) still holds for some range of $(\lambda, \mu)$ when the factor $\frac{c}{a} \frac{b}{d}$ is either greater or equal (but close enough) than unity. In [12] can be found additional details about the local behaviour of the curves $\lambda=\sigma_{1}\left[b \theta_{\mu}[d]\right]$ and $\mu=\sigma_{1}\left[c \theta_{\lambda}[a]\right]$ near $(\lambda, \mu)=\left(\sigma_{1}[0], \sigma_{1}[0]\right)$.

3.2 Building up coexistence wedges. Condition (3.8) is easily computable in terms of the several parameters appearing in (3.4). The principal eigenvalue $\sigma_{1}[0]$ can be approximated, if necessary, by using some numerical method. Similarly, from Theorem 2.3 and Theorem 3.1 we can obtain some simple sufficient conditions for the existence of a coexistence state. Given a continuous function $f: \bar{\Omega} \rightarrow \mathbb{R}$ we shall denote $f_{L} \equiv \min _{\bar{\Omega}} f$ and $f_{S} \equiv \max _{\bar{\Omega}} f$.

Theorem 3.4. Let $M_{1} \equiv M\left(d_{1}, q_{1}, \ell_{2}, A, \Omega\right), M_{2} \equiv M\left(d_{2}, q_{2}, m_{2}, D, \Omega\right)$ be two positive constants satisfying part (c) of Theorem 2.3. Assume

$$
\lambda>\sigma_{1,1} \equiv \sigma_{1}\left[d_{1}, q_{1}, \ell_{2}\right], \quad \mu>\sigma_{1,2} \equiv \sigma_{1}\left[d_{2}, q_{2}, m_{2}\right],
$$

$$
\lambda>\sigma_{1,1}+\left(\frac{B(x)}{\ell_{2}(x)}\right)_{S} M_{2}\left(\mu-\sigma_{1,2}\right), \quad \mu>\sigma_{1,2}+\left(\frac{C(x)}{m_{2}(x)}\right)_{S} M_{1}\left(\lambda-\sigma_{1,1}\right) .
$$


Then (2.2) has an asymptotically stable coexistence state.

Proof. Due to part (c) of Theorem 2.3

$$
\left(\bar{\theta}_{\lambda}\right)_{S} \leq M_{1}\left(\lambda-\sigma_{1,1}\right), \quad\left(\bar{\theta}_{\mu}\right)_{S} \leq M_{2}\left(\mu-\sigma_{1,2}\right) .
$$

Thus, from Lemma 2.1 and the second inequality of (3.11) we find that

$$
\begin{aligned}
\sigma_{1}\left[d_{1}, q_{1}+B \bar{\theta}_{\mu}, \ell_{2}\right] & \leq \sigma_{1}\left[d_{1}, q_{1}+\left(\frac{B(x)}{\ell_{2}(x)}\right)_{S} M_{2}\left(\mu-\sigma_{1,2}\right) \ell_{2}, \ell_{2}\right] \\
& =\sigma_{1,1}+\left(\frac{B(x)}{\ell_{2}(x)}\right)_{S} M_{2}\left(\mu-\sigma_{1,2}\right)
\end{aligned}
$$

and so (3.10) implies $\lambda>\sigma_{1}\left[d_{1}, q_{1}+B \bar{\theta}_{\mu}, \ell_{2}\right]$. Similarly, (3.10) implies $\mu>$ $\sigma_{1}\left[d_{2}, q_{2}+C \bar{\theta}_{\lambda}, m_{2}\right]$. Theorem 3.1 completes the proof.

Remark 3.5. Part (b) of Theorem 3.1 shows that (3.9) is necessary for the existence of a coexistence state. The region described by (3.9) is a cone with vertex at $(\lambda, \mu)=\left(\sigma_{1,1}, \sigma_{1,2}\right)$ and edges $\lambda=\sigma_{1,1}, \mu=\sigma_{1,2}$. The set of $(\lambda, \mu)$ satisfying (3.10) is the subset of (3.9) consisting of all pairs $(\lambda, \mu)$ between the straight lines

$$
\lambda=\sigma_{1,1}+\left(\frac{B(x)}{\ell_{2}(x)}\right)_{S} M_{2}\left(\mu-\sigma_{1,2}\right), \quad \mu=\sigma_{1,2}+\left(\frac{C(x)}{m_{2}(x)}\right)_{S} M_{1}\left(\lambda-\sigma_{1,1}\right) .
$$

Observe that both lines pass through by $\left(\sigma_{1,1}, \sigma_{1,2}\right)$.

Remark 3.6. The coexistence wedge (3.10) is nonempty if, and only if,

$$
\left(\frac{B(x)}{\ell_{2}(x)}\right)_{S} \cdot\left(\frac{C(x)}{m_{2}(x)}\right)_{S} \cdot M_{1} \cdot M_{2}<1 .
$$

As $M_{1}$ and $M_{2}$ are independent of $B(x)$ and $C(x)$, (3.13) holds whenever $B(x)$, or $C(x)$, is small enough (in supremum norm).

Remark 3.7. Consider the model (3.4). Then $M_{1}=M_{1}(a, \Omega)$ and $M_{2}=$ $M_{2}(d, \Omega)$ only vary with $a, d, \Omega$, and (3.13) becomes

$$
b c<\frac{1}{M_{1} M_{2}} \text {. }
$$

If (3.14) is satisfied then the set of values $(\lambda, \mu)$ satisfying $\lambda>\sigma_{1}[0], \mu>\sigma_{1}[0]$ and

$$
\lambda>\sigma_{1}[0]+b M_{2}\left(\mu-\sigma_{1}[0]\right), \quad \mu>\sigma_{1}[0]+c M_{1}\left(\lambda-\sigma_{1}[0]\right),
$$

is the wedge between the straight lines $\lambda=\sigma_{1}[0]+b M_{2}\left(\mu-\sigma_{1}[0]\right)$ and $\mu=$ $\sigma_{1}[0]+c M_{1}\left(\lambda-\sigma_{1}[0]\right)$ with vertex at $\left(\sigma_{1}[0], \sigma_{1}[0]\right)$. For every $(\lambda, \mu)$ in this wedge (3.4) has an asymptotically stable coexistence state. This result is a substantial improvement of Theorem 4.3 in [12], where both, $b$ and $c$, were assumed to be small enough.

Remark 3.8. To the best of our knowledge, Theorem 3.4 is the first result providing us with coexistence wedges centered at $\left(\sigma_{1,1}, \sigma_{1,2}\right)$ for the case of variable coefficients. The same technique applies for periodic-parabolic systems with two species, completing some results of [17]. 
3.3 Building up extinction regions. We now show how to enlarge the noncoexistence region $\lambda \leq \sigma_{1,1}, \mu \leq \sigma_{1,2}$, thus giving complementary information about coexistence (cf. part (b) of Theorem 3.1). It has been settled in part (a) of Theorem 3.1 that every coexistence state $(u, v)$ of $(2.2)$ must satisfy the estimates (3.1). Set $\bar{\theta}_{\lambda, 0} \equiv \bar{\theta}_{\lambda}, \bar{\theta}_{\mu, 0} \equiv \bar{\theta}_{\mu}, \underline{\theta}_{\lambda, 0} \equiv \underline{\theta}_{\lambda}, \underline{\theta}_{\mu, 0} \equiv \underline{\theta}_{\mu}$ and consider the sequences $\left\{\bar{\theta}_{\lambda, n}\right\},\left\{\bar{\theta}_{\mu, n}\right\},\left\{\underline{\theta}_{\lambda, n}\right\},\left\{\underline{\theta}_{\mu, n}\right\}, n \geq 0$, defined from $\bar{\theta}_{\lambda, 0}$, $\bar{\theta}_{\mu, 0}, \underline{\theta}_{\lambda, 0}$ and $\underline{\theta}_{\mu, 0}$ by the scheme

$$
\begin{aligned}
& \overline{\bar{\theta}}_{\lambda, n+1} \equiv \theta_{\lambda}\left[d_{1}, q_{1}+B \underline{\theta}_{\mu, n}, \ell_{2}, A\right], \quad \bar{\theta}_{\mu, n+1} \equiv \theta_{\mu}\left[d_{2}, q_{2}+C \underline{\theta}_{\lambda, n}, m_{2}, D\right], \\
& \underline{\theta}_{\lambda, n+1} \equiv \theta_{\lambda}\left[d_{1}, q_{1}+B \bar{\theta}_{\mu, n+1}, \ell_{2}, A\right], \quad \underline{\theta}_{\mu, n+1} \equiv \theta_{\mu}\left[d_{2}, q_{2}+C \bar{\theta}_{\lambda, n+1}, m_{2}, D\right] .
\end{aligned}
$$

Let $(u, v)$ be a componentwise nonnegative solution couple of (2.2). Arguing as in the proof of Theorem 3.1(b) we find that $\underline{\theta}_{\lambda, 0} \leq u \leq \bar{\theta}_{\lambda, 0}$ and $\underline{\theta}_{\mu, 0} \leq v \leq$ $\bar{\theta}_{\mu, 0}$. It follows from the definition of $\bar{\theta}_{\lambda, 1}$ and $\bar{\theta}_{\mu, 1}$ that $u$ and $v$ are lower solutions of $\bar{\theta}_{\lambda, 1}$ and $\bar{\theta}_{\mu, 1}$, respectively, and hence the maximum principle implies $u \leq \bar{\theta}_{\lambda, 1}, v \leq \bar{\theta}_{\mu, 1}$. In particular, $u=0$ provided $\bar{\theta}_{\lambda, 1}=0$, which occurs iff $\lambda \leq \sigma_{1}\left[d_{1}, q_{1}+B \underline{\theta}_{\mu, 0}, \ell_{2}\right]$, and $v=0$ provided $\bar{\theta}_{\mu, 1}=0$, which occurs iff $\mu \leq \sigma_{1}\left[d_{2}, q_{2}+C \underline{\theta}_{\lambda, 0}, m_{2}\right]$. Moreover, from the definition of $\underline{\theta}_{\lambda, 1}$ and $\underline{\theta}_{\mu, 1}$, two comparisons and the maximum principle show that $\underline{\theta}_{\lambda, 1} \leq u \leq$ $\bar{\theta}_{\lambda, 1}$ and $\underline{\theta}_{\mu, 1} \leq v \leq \bar{\theta}_{\mu, 1}$. Finally, from Lemma 2.1 and Theorem 2.3 we find that $\underline{\theta}_{\lambda, 0} \leq \underline{\theta}_{\lambda, 1} \leq u \leq \bar{\theta}_{\lambda, 1} \leq \bar{\theta}_{\lambda, 0}$ and that $\underline{\theta}_{\mu, 0} \leq \underline{\theta}_{\mu, 1} \leq v \leq \bar{\theta}_{\mu, 1} \leq \bar{\theta}_{\mu, 0}$ and an induction argument shows that

$$
\begin{array}{r}
\underline{\theta}_{\lambda, n} \leq \underline{\theta}_{\lambda, n+1} \leq u \leq \bar{\theta}_{\lambda, n+1} \leq \bar{\theta}_{\lambda, n}, \quad \underline{\theta}_{\mu, n} \leq \underline{\theta}_{\mu, n+1} \leq v \leq \bar{\theta}_{\mu, n+1} \leq \bar{\theta}_{\mu, n}, \\
n \geq 0 .
\end{array}
$$

Assume $\lambda>\sigma_{1}\left[d_{1}, q_{1}+B \bar{\theta}_{\mu, 0}, \ell_{2}\right]$. Then $\underline{\theta}_{\lambda, 0} \gg 0$ and hence

$$
\sigma_{1}\left[d_{2}, q_{2}+C \underline{\theta}_{\lambda, 0}, m_{2}\right]>\sigma_{1,2} \text {. }
$$

Therefore, the extinction region given by part (b) of Theorem 3.1, $\mu \leq \sigma_{1,2}$, has been enlarged by adding the set

$$
E_{\lambda}^{0}:=\left\{(\lambda, \mu): \sigma_{1,2}<\mu \leq \sigma_{1}\left[d_{2}, q_{2}+C \underline{\theta}_{\lambda, 0}, m_{2}\right]\right\},
$$

because if $(\lambda, \mu) \in E_{\lambda}^{0}$ then $\bar{\theta}_{\mu, 1}=0$ and hence $v=0$. Similarly, if $\mu>$ $\sigma_{1}\left[d_{2}, q_{2}+C \bar{\theta}_{\lambda, 0}, m_{2}\right]$ then $\underline{\theta}_{\mu, 0} \gg 0, \sigma_{1}\left[d_{1}, q_{1}+B \underline{\theta}_{\mu, 0}, \ell_{2}\right]>\sigma_{1,1}$, and the extinction region $\lambda \leq \sigma_{1,1}$ is enlarged by adding the set

$$
E_{\mu}^{0}:=\left\{(\lambda, \mu): \sigma_{1,1}<\lambda \leq \sigma_{1}\left[d_{1}, q_{1}+B \underline{\theta}_{\mu, 0}, \ell_{2}\right]\right\},
$$

because if $(\lambda, \mu) \in E_{\mu}^{0}$ then $\bar{\theta}_{\lambda, 1}=0$ and hence $u=0$. The following result provides us with the structure of $E_{\lambda}^{0}$ and $E_{\mu}^{0}$.

Lemma 3.9. The relation

$$
\lambda=\sigma_{1}\left[d_{1}, q_{1}+B \underline{\theta}_{\mu, 0}, \ell_{2}\right]
$$

defines $\lambda$ as a smooth increasing function of $\mu$, for $\mu \geq \sigma_{1,2}$, say $\lambda=\lambda_{0}(\mu)$. Similarly,

$$
\mu=\sigma_{1}\left[d_{2}, q_{2}+C \underline{\theta}_{\lambda, 0}, m_{2}\right]
$$


defines $\mu$ as a smooth increasing function of $\lambda$, for $\lambda \geq \sigma_{1,1}$, say $\mu=\mu_{0}(\lambda)$.

Proof. Denote $g(\lambda, \mu)=\sigma_{1}\left[d_{1}, q_{1}+B \underline{\theta}_{\mu, 0}, \ell_{2}\right]$. If $\mu=\sigma_{1,2}$, then $\bar{\theta}_{\mu, 0}=0$ and hence $\underline{\theta}_{\mu, 0}=0$. Thus $g\left(\lambda, \sigma_{1,2}\right)=\sigma_{1,1}$ and $\lambda=\sigma_{1,1}$ is the unique $\lambda$ satisfying (3.17). Now fix $\mu>\sigma_{1,2}$. If $\lambda=\sigma_{1,1}$ then $\underline{\theta}_{\lambda, 0}=\bar{\theta}_{\lambda, 0}=0$ and hence $\underline{\theta}_{\mu, 0}=\bar{\theta}_{\mu, 0} \gg 0$. Thus $g\left(\sigma_{1,1}, \mu\right)=\sigma_{1}\left[d_{1}, q_{1}+B \underline{\theta}_{\mu, 0}, \ell_{2}\right]>\sigma_{1,1}$. Moreover, $\underline{\theta}_{\mu, 0}$ decreases with $\lambda \geq \sigma_{1,1}$ and hence $g(\cdot, \mu)$ is decreasing. Thus, there exists a unique $\lambda=\lambda_{0}(\mu)$ such that $\lambda_{0}(\mu)=g\left(\lambda_{0}(\mu), \mu\right)$, i.e., solving (3.17). Furthermore, as $\frac{\partial g}{\partial \lambda} \leq 0, \frac{\partial}{\partial \lambda}(\lambda-g(\lambda, \mu))>0$ and hence the smooth character of $\lambda_{0}(\mu)$ as a function of $\mu$ follows from the Implicit Function Theorem. By symmetry, the assertion concerning with (3.18) follows.

Using the terminology introduced by Lemma 3.9

$$
\begin{aligned}
& E_{\lambda}^{0}=\left\{(\lambda, \mu): \lambda>\sigma_{1}\left[d_{1}, q_{1}+B \bar{\theta}_{\mu, 0}, \ell_{2}\right], \quad \sigma_{1,2}<\mu \leq \mu_{0}(\lambda)\right\}, \\
& E_{\mu}^{0}=\left\{(\lambda, \mu): \mu>\sigma_{1}\left[d_{2}, q_{2}+C \bar{\theta}_{\lambda, 0}, m_{2}\right], \quad \sigma_{1,1}<\lambda \leq \lambda_{0}(\mu)\right\} .
\end{aligned}
$$

The sharpening of the extinction region may be stepwise refined as we describe now. Assume $\lambda>\sigma_{1}\left[d_{1}, q_{1}+B \bar{\theta}_{\mu, 0}, \ell_{2}\right]$. Then $\underline{\theta}_{\lambda, 0} \gg 0$ and hence $\bar{\theta}_{\mu, 1} \ll$ $\bar{\theta}_{\mu, 0}$. Thus, (3.16) and Theorem 2.3 imply $\underline{\theta}_{\lambda, 1} \gg \underline{\theta}_{\lambda, 0}$ and

$$
\sigma_{1}\left[d_{2}, q_{2}+C \underline{\theta}_{\lambda, 1}, m_{2}\right]>\sigma_{1}\left[d_{2}, q_{2}+C \underline{\theta}_{\lambda, 0}, m_{2}\right] .
$$

Moreover, if $\mu \leq \sigma_{1}\left[d_{2}, q_{2}+C \underline{\theta}_{\lambda, 1}, m_{2}\right]$ then $\bar{\theta}_{\mu, 2}=0$ and hence $v=0$. Therefore, the extinction region again is enlarged by adding the set of couples $(\lambda, \mu)$ such that $\lambda>\sigma_{1}\left[d_{1}, q_{1}+B \bar{\theta}_{\mu, 0}, \ell_{2}\right]$ and

$$
\sigma_{1}\left[d_{2}, q_{2}+C \underline{\theta}_{\lambda, 0}, m_{2}\right]<\mu \leq \sigma_{1}\left[d_{2}, q_{2}+C \underline{\theta}_{\lambda, 1}, m_{2}\right],
$$

say $E_{\lambda}^{1}$. Similarly, the extinction region may be enlarged by adding the set, denoted by $E_{\mu}^{1}$, of couples $(\lambda, \mu)$ such that $\mu>\sigma_{1}\left[d_{2}, q_{2}+C \bar{\theta}_{\lambda, 0}, m_{2}\right]$ and

$$
\sigma_{1}\left[d_{1}, q_{1}+B \underline{\theta}_{\mu, 0}, \ell_{2}\right]<\lambda \leq \sigma_{1}\left[d_{1}, q_{1}+B \underline{\theta}_{\mu, 1}, \ell_{2}\right] \text {. }
$$

Arguing as in Lemma 3.9 it follows easily that $\mu=\sigma_{1}\left[d_{2}, q_{2}+C \underline{\theta}_{\lambda, 1}, m_{2}\right]$ defines to $\mu$ as a smooth increasing function of $\lambda$, say $\mu=\mu_{1}(\lambda)$ and that $\lambda=\sigma_{1}\left[d_{1}, q_{1}+B \underline{\theta}_{\mu, 1}, \ell_{2}\right]$ defines $\lambda$ as a smooth increasing function of $\mu$, say $\lambda=\lambda_{1}(\mu)$. With this terminology, the new extinction sets are given by

$$
\begin{aligned}
& E_{\lambda}^{1}=\left\{(\lambda, \mu): \lambda>\sigma_{1}\left[d_{1}, q_{1}+B \bar{\theta}_{\mu, 0}, \ell_{2}\right], \quad \mu_{0}(\lambda)<\mu \leq \mu_{1}(\lambda)\right\}, \\
& E_{\mu}^{1}=\left\{(\lambda, \mu): \mu>\sigma_{1}\left[d_{2}, q_{2}+C \bar{\theta}_{\lambda, 0}, m_{2}\right], \quad \lambda_{0}(\mu)<\lambda \leq \lambda_{1}(\mu)\right\} .
\end{aligned}
$$

Arguing recursively, a couple of increasing sequences $\left\{\lambda_{n}(\mu)\right\},\left\{\mu_{n}(\lambda)\right\}, n \geq 0$, can be constructed such that

$$
\begin{aligned}
& E_{\lambda}^{n}=\left\{(\lambda, \mu): \lambda>\sigma_{1}\left[d_{1}, q_{1}+B \bar{\theta}_{\mu, 0}, \ell_{2}\right], \quad \mu_{n-1}(\lambda)<\mu \leq \mu_{n}(\lambda)\right\}, \\
& E_{\mu}^{n}=\left\{(\lambda, \mu): \mu>\sigma_{1}\left[d_{2}, q_{2}+C \bar{\theta}_{\lambda, 0}, m_{2}\right], \quad \lambda_{n-1}(\mu)<\lambda \leq \lambda_{n}(\mu)\right\},
\end{aligned}
$$

are extinction sets for all $n \geq 0$. The curves $C_{(u, 0)}$ and $C_{(0, v)}$ provide us with bounds for the curves $\lambda=\lambda_{n}(\mu)$ and $\mu=\mu_{n}(\lambda)$, respectively. Hence they have a limit, say $\lambda=\lambda_{\infty}(\mu)$ and $\mu=\mu_{\infty}(\lambda)$. We have proven the following result. 
Theorem 3.10. If $(\lambda, \mu) \in \bigcup_{n=0}^{\infty}\left(E_{\lambda}^{n} \cup E_{\mu}^{n}\right)$ then (2.2) does not admit a coexistence state.

Remark 3.11. In general the curve $\lambda=\lambda_{\infty}(\mu)$ does not coincide with $C_{(0, v)}$. This is the case when the bifurcation to coexistence states from the semitrivial $(0, v)$-branch is supercritical in the $\mu$-axis direction. Similarly, in general $\mu=$ $\mu_{\infty}(\lambda)$ does not coincide with $C_{(u, 0)}$ (cf. [12], [14] and references therein).

3.4 An extinction result with biological implications. Consider the model

$$
\begin{aligned}
& -d_{1} \Delta u+q_{1}(x) u=\lambda \ell_{2}(x) u-A(x) u^{2}-\beta B(x) u v, \quad u, v \in U, \\
& -d_{2} \Delta v+q_{2}(x) v=\mu m_{2}(x) u-C(x) u v-D(x) u^{2},
\end{aligned}
$$

where $\beta$ is considered as a real parameter, $\beta \geq 0$. Observe that (2.2) is $(3.19)_{1}$. If $b(x)>0$ almost everywhere in $\Omega$, then the species $v$ competes against $u$ everywhere in the habitat $\Omega$ and from the biological point of view the species $v$ should drive $u$ to extinction if $\beta$ is large. The following result makes precise this biological principle.

Theorem 3.12. Assume that $\{x \in \Omega: b(x)=0\}$ has zero Lebesgue measure and

$$
\lambda>\sigma_{1}\left[d_{1}, q_{1}, \ell_{2}\right], \quad \mu>\sigma_{1}\left[d_{2}, q_{2}+C \bar{\theta}_{\lambda}, m_{2}\right] .
$$

Then there exists $\beta_{e}>0$ such that $(3.19)_{\beta}$ does not admit a coexistence state if $\beta>\beta_{e}$.

Proof. It follows from (3.20) and the definition of $\underline{\theta}_{\mu}$ that $\underline{\theta}_{\mu} \gg 0$. Thus, the mapping $\beta \rightarrow \sigma_{1}\left[d_{1}, q_{1}+\beta B \underline{\theta}_{\mu}, \ell_{2}\right]$ is strictly increasing. It is enough to prove that there exists $\beta>0$ (unique) such that

$$
\lambda=\sigma_{1}\left[d_{1}, q_{1}+\beta B \underline{\theta}_{\mu}, \ell_{2}\right]
$$

because then $(\lambda, \mu) \in E_{\mu}^{0}$ (cf. $\S 3.3$ ) and hence the conclusion follows. Let $K>0$ be large enough such that $-d_{1} \Delta+q_{1}+K \ell_{2}$ is coercive and

$$
(\lambda+K)\left(\ell_{2}\right)_{L}-\beta\left(B \underline{\theta}_{\mu}\right)_{S}>0 .
$$

If, for instance, $K+\sigma_{1,1}>1$ then $-d_{1} \Delta+q_{1}+K \ell_{2}$ is coercive. The operator $T_{\beta} \equiv\left[-d_{1} \Delta+q_{1}+K \ell_{2}\right]^{-1}\left[(\lambda+K) \ell_{2}-\beta B \underline{\theta}_{\mu}\right]$ is well defined and it is compact strongly order preserving. An easy calculation shows that (3.21) holds if, and only if, $\operatorname{spr} T_{\beta}=1$. We claim that $\operatorname{spr} T_{\beta}>1$ if, and only if,

$$
\lambda>\sigma_{1}\left[d_{1}, q_{1}+\beta B \underline{\theta}_{\mu}, \ell_{2}\right],
$$

and that $\operatorname{spr} T_{\beta}<1$ if, and only if, $\lambda<\sigma_{1}\left[d_{1}, q_{1}+\beta B \underline{\theta}_{\mu}, \ell_{2}\right]$. This completes the proof. To show the previous claim we first show that when $\operatorname{spr} T_{\beta}<1$, or spr $T_{\beta}>1$, it is independent for $K$ satisfying (3.22). Let $\varphi, \psi$ be unique positive functions such that

$$
\begin{array}{ll}
\left(-d_{1} \Delta+q_{1}+\beta B \underline{\theta}_{\mu}\right) \varphi=\sigma_{1}\left[d_{1}, q_{1}+\beta B \underline{\theta}_{\mu}, \ell_{2}\right] \ell_{2} \varphi, & \varphi \in U, \\
\left(-d_{1} \Delta+q_{1}+K \ell_{2}\right) \psi=\frac{1}{\operatorname{spr} T_{\beta}}\left[(\lambda+K) \ell_{2}-\beta B \underline{\theta}_{\mu}\right] \psi, & \psi \in U
\end{array}
$$


Multiplying (3.24) by $\varphi,(3.23)$ by $\psi$ and integrating on $\Omega$ we find that

$$
\operatorname{spr} T_{\beta}=\frac{(\lambda+K) \int_{\Omega} \ell_{2} \psi \varphi-\beta \int_{\Omega} B \underline{\theta}_{\mu} \psi \varphi}{\left(\sigma_{1}\left[d_{1}, q_{1}+\beta B \underline{\theta}_{\mu}, \ell_{2}\right]+K\right) \int_{\Omega} \ell_{2} \psi \varphi-\beta \int_{\Omega} B \underline{\theta}_{\mu} \psi \varphi},
$$

which shows the independence on $K$. In particular, $\operatorname{spr} T(0)=\frac{K+\lambda}{K+\sigma_{1,1}}>1$, because $\lambda>\sigma_{1,1}$ (see (3.20)). Moreover, for fixed $K>0$ satisfying (3.22), the mapping $\beta \rightarrow(\lambda+K) \ell_{2}-\beta B \underline{\theta}_{\mu}$ is strictly decreasing and so $\beta \rightarrow \operatorname{spr} T_{\beta}$ is strictly decreasing in $\beta$ as long as (3.22) is satisfied (cf. Theorem 3.2 in [1]). Thus, we only have to show that $\operatorname{spr} T_{\beta}<1$ for $\beta$ large. To simplify the notation we denote $K_{1}=K+\sigma_{1,1}$ and $L_{K}=\left[-d_{1} \Delta+q_{1}+K \ell_{2}\right]^{-1}$. As $\left(\lambda-\sigma_{1,1}+1\right) \ell_{2}-\beta B \underline{\theta}_{\mu} \leq\left[\left(\lambda-\sigma_{1,1}+1\right) \ell_{2}-\beta B \underline{\theta}_{\mu}\right]^{+}$(the positive part), we find that $\operatorname{spr} T_{\beta} \leq \operatorname{spr} L_{K}\left[\left(K_{1}-1\right) \ell_{2}+\left[\left(\lambda-\sigma_{1,1}+1\right) \ell_{2}-\beta B \underline{\theta}_{\mu}\right]^{+}\right]$. Thus, we only have to prove that $\operatorname{spr} L_{K}\left[\left(K_{1}-1\right) \ell_{2}+\left[\left(\lambda-\sigma_{1,1}+1\right) \ell_{2}-\beta B \underline{\theta}_{\mu}\right]^{+}\right]$ $<1$ if $\beta$ is large. As $\{x \in \Omega: b(x)=0\}$ has zero Lebesgue measure, $\lim _{\beta \rightarrow \infty}\left[\left(\lambda-\sigma_{1,1}+1\right) \ell_{2}-\beta B \underline{\theta}_{\mu}\right]^{+}=0$, in $L^{p}(\Omega)$ for every $p \in(1, \infty)$ (see (2.3)). Thus, arguing as in [10], Proposition 1,

$$
\lim _{\beta \rightarrow \infty} \operatorname{spr} L_{K}\left[\left(K_{1}-1\right) \ell_{2}+\left[\left(\lambda-\sigma_{1,1}+1\right) \ell_{2}-\beta B \underline{\theta}_{\mu}\right]^{+}\right]=\operatorname{spr} L_{K}\left[\left(K_{1}-1\right) \ell_{2} \cdot\right] \text {. }
$$

Finally, an easy calculation shows that $\operatorname{spr} L_{K}\left[\left(K_{1}-1\right) \ell_{2} \cdot\right]=\frac{K_{1}-1}{K_{1}}<1$, which completes the proof.

Remark 3.13. Assume $\mu>\sigma_{1,2}$. Then $\bar{\theta}_{\mu} \gg 0$ and $\beta \rightarrow \sigma_{1}\left[d_{1}, q_{1}+\beta B \bar{\theta}_{\mu}, \ell_{2}\right]$ is strictly increasing for $\beta \geq 0$. Moreover, arguing as in the previous proof we conclude that there exists a unique $\beta_{s}>0$ such that $\lambda=\sigma_{1}\left[d_{1}, q_{1}+\beta_{s} B \bar{\theta}_{\mu}, \ell_{2}\right]$. In particular, it follows from Theorem 3.1 that $(3.19)_{\beta}$ has an asymptotically stable coexistence state if $\beta<\beta_{s}$. As Theorem 3.12 establishes that $(3.19)_{\beta}$ does not admit a coexistence state if $\beta \geq \beta_{e}$, necessarily $\beta_{s} \leq \beta_{e}$. Observe that $(3.19)_{0}$ is uncoupled and so it has a coexistence state iff $(3.20)$ holds.

3.5 A new biological principle. When $b(x)$ vanishes on an open subdomain of $\Omega$ the phenomenology described by Theorem 3.12 changes drastically. Let $\Omega_{1} \subset \Omega$ be an open subdomain of $\Omega$ such that $b(x)=0$ for every $x \in \Omega_{1}$. Then, even if $\beta$ is large, the species $u$ may survive, because it is free of $v$ on $\Omega_{1}$. The following result makes precise this fact.

Theorem 3.14. Let $\Omega_{1} \subset \Omega$ be a nonempty subdomain such that $b(x)=0$ for every $x \in \Omega_{1}$. Let $\sigma_{1}^{\Omega_{1}}\left[d_{1}, q_{1}, \ell_{2}\right]$ be the principal eigenvalue of

$$
\left(-d_{1} \Delta+q_{1}\right) \varphi=\sigma \ell_{2} \varphi, \text { in } \Omega_{1},\left.\quad \varphi\right|_{\partial \Omega_{1}}=0 .
$$

Assume

$$
\lambda>\sigma_{1}^{\Omega_{1}}\left[d_{1}, q_{1}, \ell_{2}\right], \quad \mu>\sigma_{1}\left[d_{2}, q_{2}+C \bar{\theta}_{\lambda}, m_{2}\right] .
$$

Then (3.19) $)_{\beta}$ has an asymptotically stable coexistence state for every $\beta \geq 0$.

Proof. As $\Omega_{1} \subset \Omega, \sigma_{1}^{\Omega_{1}}\left[d_{1}, q_{1}, \ell_{2}\right] \geq \sigma_{1,1}$ and hence (3.25) implies (3.20). We now show that

$$
\sigma_{1}^{\Omega_{1}}\left[d_{1}, q_{1}, \ell_{2}\right]>\sigma_{1}\left[d_{1}, q_{1}+\beta B \bar{\theta}_{\mu}, \ell_{2}\right], \quad \beta \geq 0 .
$$


Let $\varphi$ be the unique positive function (up to multiplicative constants) satisfying

$$
\left(-d_{1} \Delta+q_{1}+\beta B \bar{\theta}_{\mu}\right) \varphi=\sigma_{1}\left[d_{1}, q_{1}+\beta B \bar{\theta}_{\mu}, \ell_{2}\right] \ell_{2} \varphi,\left.\quad \varphi\right|_{\partial \Omega}=0 .
$$

Then $\left(-d_{1} \Delta+q_{1}-\sigma_{1}\left[d_{1}, q_{1}+\beta B \bar{\theta}_{\mu}, \ell_{2}\right] \ell_{2}\right) \varphi=0$ in $\Omega_{1}$ and $\left.\varphi\right|_{\partial \Omega_{1}}>0$. Thus, the maximum principle implies $\sigma_{1}^{\Omega_{1}}\left[d_{1}, q_{1}-\sigma_{1}\left[d_{1}, q_{1}+\beta B \bar{\theta}_{\mu}, \ell_{2}\right] \ell_{2}, 1\right]>0$. On the other hand, it follows from Lemma 2.1 that

$$
\begin{aligned}
\sigma_{1}^{\Omega_{1}} & {\left[d_{1}, q_{1}-\sigma_{1}\left[d_{1}, q_{1}+\beta B \bar{\theta}_{\mu}, \ell_{2}\right] \ell_{2}, \ell_{2}\right] } \\
& >\sigma_{1}^{\Omega_{1}}\left[d_{1}, q_{1}-\sigma_{1}\left[d_{1}, q_{1}+\beta B \bar{\theta}_{\mu}, \ell_{2}\right] \ell_{2},\left(\ell_{2}\right)_{S}\right] \\
& =\left(\ell_{2}\right)_{S}^{-1} \sigma_{1}^{\Omega_{1}}\left[d_{1}, q_{1}-\sigma_{1}\left[d_{1}, q_{1}+\beta B \bar{\theta}_{\mu}, \ell_{2}\right] \ell_{2}, 1\right]>0 .
\end{aligned}
$$

Thus

$$
\begin{aligned}
& \sigma_{1}^{\Omega_{1}}\left[d_{1}, q_{1}-\sigma_{1}\left[d_{1}, q_{1}+\beta B \bar{\theta}_{\mu}, \ell_{2}\right] \ell_{2}, \ell_{2}\right] \\
& \quad=\sigma_{1}^{\Omega_{1}}\left[d_{1}, q_{1}, \ell_{2}\right]-\sigma_{1}\left[d_{1}, q_{1}+\beta B \bar{\theta}_{\mu}, \ell_{2}\right]>0,
\end{aligned}
$$

which is (3.26). As (3.25) and (3.26) imply (3.2) for every $\beta \geq 0$, Theorem 3.1 concludes the proof.

Remark 3.15. Let $\Omega^{*}$ be the interior of $\{x \in \Omega: b(x)=0\}$. Then

$$
\lim _{\beta \rightarrow \infty}\left[\left(\lambda-\sigma_{1,1}+1\right) \ell_{2}-\beta B \underline{\theta}_{\mu}\right]^{+}=\left(\lambda-\sigma_{1,1}+1\right) \ell_{2} \chi_{\Omega^{*}},
$$

in $L^{p}(\Omega)$, for every $p \in(1, \infty)$, where $\chi_{\Omega^{*}}$ is the characteristic function of $\Omega^{*}$. Thus

$$
\begin{aligned}
& \lim _{\beta \rightarrow \infty} \operatorname{spr} L_{K}\left[\left(K+\sigma_{1,1}-1\right) \ell_{2}+\left[\left(\lambda-\sigma_{1,1}+1\right) \ell_{2}-\beta B \underline{\theta}_{\mu}\right]^{+}\right] \\
& =\operatorname{spr} L_{K}\left[\left(K+\sigma_{1,1}-1\right) \ell_{2}+\left(\lambda-\sigma_{1,1}+1\right) \ell_{2} \chi_{\Omega^{*}}\right],
\end{aligned}
$$

and therefore whenever (3.25) holds it follows from Theorem 3.14 that

$$
\operatorname{spr} L_{K}\left[\left(K+\sigma_{1,1}-1\right) \ell_{2}+\left(\lambda-\sigma_{1,1}+1\right) \ell_{2} \chi_{\Omega^{*}}\right]>1,
$$

which is the converse of the result obtained in the proof of Theorem 3.12.

\section{Global attractivity of Coexistence states}

First we shall obtain an attractivity result concerning with the evolutionary model (2.1). The result says that under condition (3.2) if (2.2) has exactly one coexistence state then it attracts to every solution of $(2.1)$ having positive initial data. A sufficient condition for uniqueness will be given also. As a consequence of these results we obtain the following biological principle: Under condition (3.2) if $B(x)$, or $C(x)$, is small enough then no species can drive the other to extinction. In Section 9 we shall show that the sufficient condition for uniqueness is also necessary.

To put the attractivity theorem in its right place some preliminary results are needed. Consider the initial boundary value problem, which is equivalent to 
$(1.2)$

$$
\begin{gathered}
\frac{\partial u}{\partial t}-d_{1} \Delta u+q_{1}(x) u=\lambda \ell_{2}(x) u-A(x) u^{2}-B(x) u v, \\
(x, t) \in \Omega \times(0, T), \\
\frac{\partial v}{\partial t}-d_{2} \Delta v+q_{2}(x) v=\mu m_{2}(x) v-C(x) u v-D(x) v^{2}, \\
(x, t) \in \Omega \times(0, T), \\
(u(\cdot, 0), v(\cdot, 0))=\left(u_{0}, v_{0}\right),\left.\quad u\right|_{\partial \Omega}=\left.v\right|_{\partial \Omega}=0, t \in(0, T),
\end{gathered}
$$

where the coefficients satisfy the same hypotheses as in the previous sections, $u_{0}, v_{0} \in C^{\nu}(\bar{\Omega})$, and $T>0$. Let $R \subset C^{\nu}(\bar{\Omega}) \times C^{\nu}(\bar{\Omega})$ be the set of couples $(u, v)$ satisfying $(3.1)$ and pick $\left(u_{0}, v_{0}\right) \in R$ such that $u_{0}=v_{0}=0$ on $\partial \Omega$. Then it is well known that $(4.1)$ has a unique classical solution $\left(u\left(x, t, u_{0}, v_{0}\right), v\left(x, t, u_{0}, v_{0}\right)\right) \in C^{2+\nu}(\Omega \times(0, \infty)) \cap C(\bar{\Omega} \times[0, \infty)),[13]$, such that $\left(u\left(\cdot, t, u_{0}, v_{0}\right), v\left(\cdot, t, u_{0}, v_{0}\right)\right) \in R$ for all $t \in[0, \infty)$. See [20], [21], [25], [26], [30] and also [29], for related ideas in scalar equations. More generally, the functions defining the invariant rectangle $R$ may be replaced by other adequate upper and lower solutions, [26].

Assume that (3.2) is satisfied and that (2.2) has a unique coexistence state, $(u, v)$. It follows from Theorem 3.1 that $(u, v) \in R$. Moreover, if $\left(u_{0}, v_{0}\right) \in$ $R$ then the solution $\left(u\left(x, t, u_{0}, v_{0}\right), v\left(x, t, u_{0}, v_{0}\right)\right)$ is attracted by $(u(x)$, $v(x))$. More precisely,

$(4.2)$

$$
\lim _{t \rightarrow \infty}\left\|u\left(\cdot, t, u_{0}, v_{0}\right)-u(\cdot)\right\|_{\infty, \Omega}=0, \quad \lim _{t \rightarrow \infty}\left\|v\left(\cdot, t, u_{0}, v_{0}\right)-v(\cdot)\right\|_{\infty, \Omega}=0
$$

This behaviour is well known in the context of scalar equations, [29], and can be extended to a class of competition-type systems including (4.1), [6] and [17]. The attractivity result in this section guarantees that under condition (3.2) the uniqueness of $(u, v)$ also entails its global attractive character for all solutions with nonnegative nontrivial initial data $\left(u_{0}, v_{0}\right)$. In other words, (4.2) holds provided $u_{0}>0, v_{0}>0$. To the best of our knowledge the only available result of this nature is Theorem 4.1 in [6], valid for the system

$$
\frac{\partial u}{\partial t}=\Delta u+\lambda u-a u^{2}-b u v, \quad \frac{\partial v}{\partial t}=\Delta v+\mu v-c u v-d v^{2}
$$

under condition (3.8). The following result is a substantial improvement of Theorem 4.1 of [6].

Theorem 4.1. Assume that (3.2) is satisfied and that (2.2) has a unique coexistence state, $(u(x), v(x))$. Then for every pair of initial data $\left(u_{0}, v_{0}\right) \in$ $C^{\nu}(\bar{\Omega}) \times C^{\nu}(\bar{\Omega})$, such that $u_{0}>0, v_{0}>0$ and $u_{0}=v_{0}=0$ on $\partial \Omega$, the solution $\left(u\left(x, t, u_{0}, v_{0}\right), v\left(x, t, u_{0}, v_{0}\right)\right)$ of $(4.1)$ converges to $(u(x), v(x))$, as $t \rightarrow \infty$, uniformly in $\bar{\Omega}$.

Proof. Let $\left(u_{0}, v_{0}\right) \in C^{\nu}(\bar{\Omega}) \times C^{\nu}(\bar{\Omega})$ be such that $u_{0}>0, v_{0}>0$ and $u_{0}=$ $v_{0}=0$ on $\partial \Omega$. The existence of a unique classical solution $\left(u\left(x, t, u_{0}, v_{0}\right)\right.$, $\left.v\left(x, t, u_{0}, v_{0}\right)\right)$ of $(4.1)$ componentwise nonnegative follows readily by choosing suitable pairs $(\underline{u}, \bar{v}),(\bar{u}, \underline{v})$ of lower and upper solutions (cf. the proof of 
Theorem 3.1 and [17], [25], [29]). For the present case we can take $\underline{u}=\underline{v}=0$,

$$
\begin{aligned}
& \bar{u}=\max \left\{\left\|u_{0}\right\|_{\infty} \frac{\lambda\left\|\ell_{2}\right\|_{\infty}-\delta_{1} \min _{\bar{\Omega}} q_{1}}{\min _{\bar{\Omega}} A}\right\}, \\
& \bar{v}=\max \left\{\left\|v_{0}\right\|_{\infty}, \frac{\left.\mu\left\|m_{2}\right\|_{\infty}-\delta_{2} \min _{\bar{\Omega}} q_{2}\right)}{\min _{\bar{\Omega}} D}\right\},
\end{aligned}
$$

where $\delta_{1}=0$ if $\min _{\bar{\Omega}} q_{1} \leq 0$ and $\delta_{1}=1$ otherwise, with the same convention for $\delta_{2}$. Moreover, $\left(u\left(x, t, u_{0}, v_{0}\right), v\left(x, t, u_{0}, v_{0}\right)\right)$ can be prolonged to any time $t \geq 0$.

We now show that $u\left(x, t, u_{0}, v_{0}\right)>0, v\left(x, t, u_{0}, v_{0}\right)>0$ for every $(x, t) \in \Omega \times(0, \infty)$. To this we argue by contradiction assuming that $u\left(x_{1}, t_{1}, u_{0}, v_{0}\right)=0$ for some $\left(x_{1}, t_{1}\right) \in \Omega \times(0, \infty)$. Observe that $z(x, t)$ $\equiv u\left(x, t, u_{0}, v_{0}\right)$ is the solution of the parabolic problem,

$$
\begin{gathered}
\frac{\partial z}{\partial t}-d_{1} \Delta z+\left(k_{0}+q_{1}(x)+A(x) u+B(x) v\right) z=\left(\lambda \ell_{2}+k_{0}\right) z, \\
z(\cdot, 0)=u_{0},\left.\quad z\right|_{\partial \Omega}=0, t>0,
\end{gathered}
$$

where $k_{0}$ is taken large enough so that

$$
\min _{\bar{\Omega}}\left(k_{0}+q_{1}(x)\right) \geq 0 \text { and } \min _{\bar{\Omega}}\left(\lambda \ell_{2}+k_{0}\right) \geq 0 .
$$

Then $\frac{\partial z}{\partial t}-d_{1} \Delta z+r(x, t) z \geq 0$ in $\Omega \times(0, \infty)$, where $r(x, t)$ is the nonnegative coefficient of $z(x, t)$ in the left-hand side of the differential equation of (4.3), and hence from the parabolic strong maximum principle, [27], we find that $z(x, t)=u\left(x, t, u_{0}, v_{0}\right)=0$ for every $(x, t) \in \Omega \times\left[0, t_{1}\right]$. This contradicts $u_{0}>0$ and therefore $u\left(x, t, u_{0}, v_{0}\right)>0$ for every $(x, t) \in \Omega \times(0, \infty)$. Moreover, $\frac{\partial u}{\partial n}\left(\cdot, t, u_{0}, v_{0}\right)<0$ at $\partial \Omega$ for every $t>0$, where $n$ is the outward unit normal to $\Omega$ at $\partial \Omega$. By symmetry, the same features hold for $v\left(x, t, u_{0}, v_{0}\right)$.

We now show that the flow $\left(u\left(x, t, u_{0}, v_{0}\right), v\left(x, t, u_{0}, v_{0}\right)\right)$ defined by (4.1) is dissipative in $C^{\nu}(\bar{\Omega}) \times C^{\nu}(\bar{\Omega})$. Roughly speaking, this means that $\left(u\left(x, t, u_{0}, v_{0}\right), v\left(x, t, u_{0}, v_{0}\right)\right)$ enters into bounded regions $B$ of $C^{\nu}(\bar{\Omega}) \times$ $C^{\nu}(\bar{\Omega})$ after some finite time $t(B) \geq 0$. In fact, the information we will obtain is more precise. Fix $t_{1}>0$ and regard $\left(u_{01}, v_{01}\right)=\left(u\left(\cdot, t_{1}, u_{0}, v_{0}\right)\right.$, $\left.v\left(\cdot, t_{1}, u_{0}, v_{0}\right)\right)$ as an initial data of (4.1). By uniqueness we find that $u\left(x, t, u_{01}, v_{01}\right)=u\left(x, t+t_{1}, u_{0}, v_{0}\right)$ and that $v\left(x, t, u_{01}, v_{01}\right)=$ $v\left(x, t+t_{1}, u_{0}, v_{0}\right)$. Moreover, since $u_{01}(x)>0, v_{01}(x)>0, x \in \Omega$, and $\frac{\partial u_{01}}{\partial n}<0, \frac{\partial v_{01}}{\partial n}<0$ on $\partial \Omega$, there exists a constant $k_{1} \geq 1$ such that $u_{01}<k_{1} \bar{\theta}_{\lambda}$, $v_{01}<k_{1} \bar{\theta}_{\mu}$, in $\Omega$, and $\frac{\partial\left(u_{01}-k_{1} \bar{\theta}_{\lambda}\right)}{\partial n}>0, \frac{\partial\left(v_{01}-k_{1} \bar{\theta}_{\mu}\right)}{\partial n}>0$, on $\partial \Omega$. On the other hand, since the function $\bar{z}_{1}=k_{1} \bar{\theta}_{\lambda}$ is an upper solution of

$$
-d_{1} \Delta z_{1}+q_{1}(x) z_{1}=\lambda \ell_{2} z_{1}-A(x) z_{1}^{2},\left.\quad z_{1}\right|_{\partial \Omega} \equiv 0,
$$

it is well known, [29], that the unique classical solution of

$$
\begin{gathered}
\frac{\partial Z_{1}}{\partial t}-d_{1} \Delta Z_{1}+q_{1}(x) Z_{1}=\lambda \ell_{2} Z_{1}-A(x) Z_{1}^{2}, \quad(x, t) \in \Omega \times(0, \infty), \\
Z_{1}(\cdot, 0)=k_{1} \bar{\theta}_{\lambda},\left.\quad Z_{1}\right|_{\partial \Omega}=0, t>0,
\end{gathered}
$$

is decreasing in $t$, is defined in the whole cylinder $\bar{\Omega} \times[0, \infty)$ and $\lim _{t \rightarrow \infty} Z_{1}(x, t)$ $=\bar{\theta}_{\lambda}$ in $C^{1}(\bar{\Omega})$. This construction shows that $u_{1}(x, t) \equiv u\left(x, t, u_{01}, v_{01}\right)$ 
satisfies

$$
\begin{gathered}
\frac{\partial u_{1}}{\partial t}-d_{1} \Delta u_{1}+q_{1}(x) u_{1} \leq \lambda \ell_{2} u_{1}-A(x) u_{1}^{2}, \quad(x, t) \in \Omega \times(0, \infty), \\
u_{1}(\cdot, 0)=u_{01} \leq Z_{1}(\cdot, 0),\left.\quad u_{1}\right|_{\partial \Omega}=0, t>0,
\end{gathered}
$$

and hence the parabolic maximum principle implies

$$
u_{1}(x, t) \leq Z_{1}(x, t), \quad(x, t) \in \bar{\Omega} \times[0, \infty) .
$$

Similarly, $v_{1}(x, t) \equiv v\left(x, t, u_{01}, v_{01}\right)$ satisfies

$$
v_{1}(x, t) \leq Z_{2}(x, t), \quad(x, t) \in \bar{\Omega} \times[0, \infty),
$$

where $Z_{2}=Z_{2}(x, t)$ is the unique solution of

$$
\begin{aligned}
\frac{\partial Z_{2}}{\partial t}-d_{2} \Delta Z_{2}+q_{2}(x) Z_{2} & =\mu m_{2} Z_{2}-D(x) Z_{2}^{2}, \quad(x, t) \in \Omega \times(0, \infty), \\
Z_{2}(\cdot, 0) & =k_{1} \bar{\theta}_{\mu},\left.\quad Z_{2}\right|_{\partial \Omega}=0, \quad t>0 .
\end{aligned}
$$

As $Z_{1}(x, t), Z_{2}(x, t)$ is decreasing in $t$ and $\lim _{t \rightarrow \infty} Z_{2}(x, t)=\bar{\theta}_{\mu}$ in $C^{1}(\bar{\Omega})$. The estimates (4.4) and (4.5) show the dissipative character of the flow generated by (4.1) in $C(\bar{\Omega}) \times C(\bar{\Omega})$, [16]. Some well-known a priori estimates show the dissipative character of the flow in $C^{\nu}(\bar{\Omega}) \times C^{\nu}(\bar{\Omega}),[13]$.

The last step of the proof consists in showing the existence of suitable pairs of lower and upper solutions $(\underline{u}(x), \bar{v}(x)),(\bar{u}(x), \underline{v}(x))$ (see [26]) and a time $t_{2} \geq t_{1}$ such that

$$
0<\underline{u}(x) \leq u\left(x, t_{2}, u_{0}, v_{0}\right) \leq \bar{u}(x), \quad 0<\underline{v}(x) \leq v\left(x, t_{2}, u_{0}, v_{0}\right) \leq \bar{v}(x)
$$

in $\Omega$. Then, the general theory of lower and upper solutions extended to the present case shows that the solution of (4.1)

$$
\left(u_{2}(x, t), v_{2}(x, t)\right) \equiv\left(u\left(x, t, u_{02}, v_{02}\right), v\left(x, t, u_{02}, v_{02}\right)\right)
$$

with initial data $\left(u_{02}, v_{02}\right)=\left(u\left(\cdot, t_{2}, u_{0}, v_{0}\right), v\left(\cdot, t_{2}, u_{0}, v_{0}\right)\right)$ satisfies

$$
\lim _{t \rightarrow \infty}\left(u_{2}(\cdot, t), v_{2}(\cdot, t)\right)=(u, v),
$$

uniformly in $\bar{\Omega}$, because $(u, v)$ is the unique coexistence state of $(2.2)$. Observe that the existence of such couples of lower and upper solutions for the elliptic model implies the existence of some coexistence state in between them. This follows arguing as in the proof of Theorem 3.1. In fact, condition (3.2) allowed us to construct such couples. Moreover, as we are assuming that $(u, v)$ is the unique coexistence state of (2.2), $\underline{u} \leq u \leq \bar{u}$ and $\underline{v} \leq v \leq \bar{v}$. Finally, since $\left(u_{2}(\cdot, t), v_{2}(\cdot, t)\right)=\left(u\left(\cdot, t+t_{2}, u_{0}, v_{0}\right), v\left(\cdot, t+t_{2}, u_{0}, v_{0}\right)\right)$, it follows from (4.7) that $\lim _{t \rightarrow \infty}\left(u\left(x, t, u_{0}, v_{0}\right), v\left(x, t, u_{0}, v_{0}\right)\right)=(u(x), v(x))$ uniformly in $\bar{\Omega}$, which completes the proof. To construct $\underline{u}, \bar{u}, \underline{v}$ and $\bar{v}$, pick $(\lambda, \mu)$ satisfying (3.2), take a pair $\left(\lambda_{1}, \mu_{1}\right)$ such that $\lambda<\lambda_{1}<g^{-1}(\mu), \mu<$ $\mu_{1}<h^{-1}(\lambda)$, where $h(\mu)=\sigma_{1}\left[d_{1}, q_{1}+B \bar{\theta}_{\mu}, \ell_{2}\right], g(\lambda)=\sigma_{1}\left[d_{2}, q_{2}+C \bar{\theta}_{\lambda}, m_{2}\right]$ and define

$$
\bar{u}=\theta_{\lambda_{1}}\left[d_{1}, q_{1}, \ell_{2}, A\right], \quad \bar{v}=\theta_{\mu_{1}}\left[d_{2}, q_{2}, m_{2}, D\right]
$$

Then

$-d_{1} \Delta \bar{u}+q_{1}(x) \bar{u}=\lambda_{1} \ell_{2} \bar{u}-A(x) \bar{u}^{2} \geq \lambda \ell_{2} \bar{u}-A(x) \bar{u}^{2} \geq \lambda \ell_{2} \bar{u}-A(x) \bar{u}^{2}-B(x) \bar{u} \underline{v}$, 
where $\underline{v}$ is a positive function to be chosen later. Similarly,

$$
-d_{2} \Delta \bar{v}+q_{2}(x) \bar{v}=\mu_{1} m_{2} \bar{v}-D(x) \bar{v}^{2} \geq \mu m_{2} \bar{v}-D(x) \bar{v}^{2}-C(x) \underline{u} \bar{v},
$$

where $\underline{u}$ is positive function to be chosen later. As $\mu_{1}<h^{-1}(\lambda), \lambda>$ $\sigma_{1}\left[d_{1}, q_{1}+B \bar{v}, \ell_{2}\right]$ and hence

$$
\varepsilon A(x) \varphi_{1}\left[d_{1}, q_{1}+B \bar{v}, \ell_{2}\right] \leq \lambda-\sigma_{1}\left[d_{1}, q_{1}+B \bar{v}, \ell_{2}\right],
$$

for $\varepsilon>0$ small. Let $\varepsilon>0$ be one of such values and define $\underline{u} \equiv \varepsilon \varphi_{1}\left[d_{1}, q_{1}+\right.$ $\left.B \bar{v}, \ell_{2}\right]$. Then

$-d_{1} \Delta \underline{u}+q_{1} \underline{u}-\lambda \ell_{2} \underline{u}+A \underline{u}^{2}+B \underline{u} \bar{v}=-\left(\lambda-\sigma_{1}\left[d_{1}, q_{1}+C \bar{v}, \ell_{2}\right]\right) \varepsilon \varphi_{1}+\varepsilon^{2} A \varphi_{1}^{2} \leq 0$.

and so $\underline{u}$ is a lower solution, for any $\varepsilon>0$ small enough. Similarly, it follows from $\lambda_{1}<g^{-1}(\mu)$ that $\underline{v} \equiv \varepsilon \varphi_{1}\left[d_{2}, q_{2}+C \bar{u}, m_{2}\right]$ is a lower solution provided

$$
\varepsilon D(x) \varphi_{1}\left[d_{2}, q_{2}+C \bar{u}, m_{2}\right] \leq \mu-\sigma_{1}\left[d_{2}, q_{2}+C \bar{u}, m_{2}\right],
$$

which is accomplished for $\varepsilon>0$ small enough. We now show that there exists $t_{2}>0$ such that (4.6) holds. As $\lambda<\lambda_{1}$, it follows from the definition of $\bar{u}$ that $\bar{\theta}_{\lambda}<\bar{u}$. Moreover, $u\left(\cdot, t, u_{0}, v_{0}\right) \leq Z_{1}(\cdot, t)$ and $\lim _{t \rightarrow \infty} Z_{1}(\cdot, t)=\bar{\theta}_{\lambda}$, in $C^{1}(\bar{\Omega})$. Thus, the upper estimates of (4.6) follow. Observe that the $v$ estimate follows as the $u$-estimate. Furthermore, $u\left(x, t_{2}, u_{0}, v_{0}\right)>0$ for every $x \in \Omega$ and $\frac{\partial u}{\partial n}\left(x, t_{2}, u_{0}, v_{0}\right)<0$ for every $x \in \partial \Omega$. Therefore, we can choose $\varepsilon$ smaller, if necessary, so that the lower estimates of (4.6) also hold. This completes the proof.

Assuming (3.20), it follows from Theorem 4.1 that the unique coexistence state of $(3.19)_{0}$ is a global attractor. We now show that the same occurs if $\beta$ is small enough, estimating the range of such $\beta$.

Theorem 4.2. Assume (3.2) and

$$
\left(\frac{B(x)}{A(x)}\right)_{S} \cdot\left(\frac{C(x)}{D(x)}\right)_{S} \cdot\left(\frac{\bar{\theta}_{\lambda}}{\underline{\theta}_{\mu}}\right)_{S} \cdot\left(\frac{\bar{\theta}_{\mu}}{\underline{\theta}_{\lambda}}\right)_{S}<1 .
$$

Then (2.2) has a unique coexistence state, which is a global attractor of solutions of (2.1) with nontrivial positive initial data.

Proof. We first show that (4.8) implies that any coexistence state of (2.2) is nondegenerate. A further continuation argument completes the proof. Let $\left(u_{0}, v_{0}\right)$ be a coexistence state of (2.2). Then the linearization of $(2.2)$ at $\left(u_{0}, v_{0}\right)$ is given by

$$
L_{1}\left[u_{0}, v_{0}\right] u=-B(x) u_{0} v, \quad L_{2}\left[u_{0}, v_{0}\right] v=-C(x) v_{0} u, \quad u, v \in U
$$

where

$$
\begin{aligned}
& L_{1}\left[u_{0}, v_{0}\right]=-d_{1} \Delta+q_{1}(x)+2 A(x) u_{0}+B(x) v_{0}-\lambda \ell_{2}, \\
& L_{2}\left[u_{0}, v_{0}\right]=-d_{2} \Delta+q_{2}(x)+2 D(x) v_{0}+C(x) u_{0}-\mu m_{2}
\end{aligned}
$$

As $\left(u_{0}, v_{0}\right)$ is a solution of equation (2.2), $\left(L_{1}\left[u_{0}, v_{0}\right]-A(x)\right) u_{0}=0$ and $\left(L_{2}\left[u_{0}, v_{0}\right]-D(x)\right) v_{0}=0$. Thus, zero is the principal eigenvalue of the elliptic operators $L_{1}\left[u_{0}, v_{0}\right]-A(x)$ and $L_{2}\left[u_{0}, v_{0}\right]-D(x)$ subject to homogeneous Dirichlet boundary conditions. Hence, the principal eigenvalues of $L_{1}\left[u_{0}, v_{0}\right]$ and $L_{2}\left[u_{0}, v_{0}\right]$ are positive, because $A(x)>0$ and $D(x)>0$, and 
so $L_{1}^{-1}\left[u_{0}, v_{0}\right]$ and $L_{2}^{-1}\left[u_{0}, v_{0}\right]$ are well defined, compact and strongly order preserving, i.e., $L_{1}\left[u_{0}, v_{0}\right]$ and $L_{2}\left[u_{0}, v_{0}\right]$ satisfy the strong maximum principle. By eliminating $u$ from the first equation of (4.9) and $v$ from the second one we find that $u=K\left[u_{0}, v_{0}\right] u$, where

$$
K\left[u_{0}, v_{0}\right]=L_{1}^{-1}\left[u_{0}, v_{0}\right]\left(B(x) u_{0} L_{2}^{-1}\left[u_{0}, v_{0}\right]\left(C(x) v_{0} \cdot\right)\right) .
$$

If $\operatorname{spr} K\left[u_{0}, v_{0}\right]<1$ then $u=0$ is the unique solution of $u=K\left[u_{0}, v_{0}\right] u$ and hence $(u, v)=(0,0)$ is the unique solution of $(4.9)$, which shows that any coexistence state of $(2.2)$ is nondegenerate. We now prove that (4.8) is sufficient for $\operatorname{spr} K\left[u_{0}, v_{0}\right]<1$. As $\operatorname{spr} K\left[u_{0}, v_{0}\right] \leq\left(\frac{B(x)}{A(x)}\right)_{S} \cdot\left(\frac{C(x)}{D(x)}\right)_{S} \cdot \operatorname{spr} T\left[u_{0}, v_{0}\right]$, where

$$
T\left[u_{0}, v_{0}\right]=L_{1}^{-1}\left[u_{0}, v_{0}\right]\left(A(x) u_{0} L_{2}^{-1}\left[u_{0}, v_{0}\right]\left(D(x) v_{0} \cdot\right)\right),
$$

we only have to show that

$$
\operatorname{spr} T\left[u_{0}, v_{0}\right]<\left(\frac{\bar{\theta}_{\lambda}}{\underline{\theta}_{\mu}}\right)_{S} \cdot\left(\frac{\bar{\theta}_{\mu}}{\underline{\theta}_{\lambda}}\right)_{S} .
$$

The operator $T\left[u_{0}, v_{0}\right]$ is compact and defines a strongly order preserving endomorphism of $C^{\nu}(\bar{\Omega})$. Moreover, (2.2) implies $L_{2}^{-1}\left[u_{0}, v_{0}\right]\left(D(x) v_{0}^{2}\right)=v_{0}$, $L_{1}^{-1}\left[u_{0}, v_{0}\right]\left(A(x) u_{0}^{2}\right)=u_{0}$. Thus,

$$
T\left[u_{0}, v_{0}\right] v_{0}=L_{1}^{-1}\left[u_{0}, v_{0}\right]\left(A(x) u_{0} v_{0}\right)<\left(\frac{v_{0}}{u_{0}}\right)_{S}\left(\frac{u_{0}}{v_{0}}\right)_{S} v_{0}
$$

and hence $\operatorname{spr} T\left[u_{0}, v_{0}\right]<\left(\frac{v_{0}}{u_{0}}\right)_{S}\left(\frac{u_{0}}{v_{0}}\right)_{S}$. As any coexistence state of (2.2) satisfies (3.1), (4.12) holds true and therefore any coexistence state of (2.2) is nondegenerate.

We now back to $(3.19)_{\beta}$ and consider $\beta$ as the main continuation parameter to show that (2.2) has a unique coexistence state. From (3.2) and (4.8) we find that

$$
\begin{gathered}
\lambda>\sigma_{1}\left[d_{1}, q_{1}(x)+\beta B(x) \bar{\theta}_{\mu}, \ell_{2}\right], \quad \mu>\sigma_{1}\left[d_{2}, q_{2}(x)+C(x) \bar{\theta}_{\lambda}, m_{2}\right], \\
\left(\frac{\beta B(x)}{A(x)}\right)_{S} \cdot\left(\frac{C(x)}{D(x)}\right)_{S} \cdot\left(\frac{\bar{\theta}_{\lambda}}{\underline{\theta}_{\mu}}\right)_{S} \cdot\left(\frac{\bar{\theta}_{\mu}}{\underline{\theta}_{\lambda, \beta}}\right)_{S}<1,
\end{gathered}
$$

for every $\beta \in[0,1]$, where $\underline{\theta}_{\lambda, \beta} \equiv \theta_{\lambda}\left[d_{1}, q_{1}(x)+\beta B(x) \bar{\theta}_{\mu}, \ell_{2}, A(x)\right]$. To get the last inequality we use that $\underline{\theta}_{\lambda, \beta} \geq \underline{\theta}_{\lambda}$ for every $\beta \leq 1$. Thus, for all $\beta \in[0,1]$ any coexistence state of $(3.19)_{\beta}$ is nondegenerate. When $\beta=0$ the system is uncoupled and so it has a unique coexistence state. Finally, an easy continuation argument in $\beta$ shows that $(3.19)_{\beta}$ has a unique coexistence state if $\beta \in[0,1],[24]$. In particular, (2.2) has a unique coexistence state. Moreover, due to Theorem 4.1 it is globally asymptotically stable.

Corollary 4.3. Assume (3.20). Then there exists $\beta_{0}>0$ such that $(3.19)_{\beta}$ has a unique coexistence state if $\beta \in\left[0, \beta_{0}\right)$. Moreover, each of these coexistence states is globally asymptotically stable.

In particular, if (3.20) is satisfied and $\|B\|_{\infty}$ is small enough then no species can drive the other to extinction. By symmetry the same result holds when $\lambda>$ 
$\sigma_{1}\left[d_{1}, q_{1}(x)+B(x) \bar{\theta}_{\mu}, \ell_{2}\right], \mu>\sigma_{1}\left[d_{2}, q_{2}(x), m_{2}\right]$ and $\|C\|_{\infty}$ is small enough. From (4.13) can be found some lower estimates for $\beta_{0}$. Such estimates involve the several coefficients of (2.2) and some geometrical constants depending on $\Omega,[14]$. When $\beta$ is large then the dynamics of $(3.19)_{\beta}$ may change drastically. In fact, if $\{x \in \Omega: b(x)=0\}$ has measure zero and $\beta>\beta_{e}$ (see Theorem 3.12) then $\left(0, \bar{\theta}_{\mu}\right)$ is a global attractor, i.e., $v$ drives $u$ to extinction. Next result makes precise this fact.

Theorem 4.4. (i) If

$$
\lambda>\sigma_{1}\left[d_{1}, q_{1}+B \bar{\theta}_{\mu}, \ell_{2}\right], \quad \mu<\sigma_{1}\left[d_{2}, q_{2}+C \bar{\theta}_{\lambda}, m_{2}\right]
$$

and (2.2) does not admit a coexistence state, then for every pair of initial data $\left(u_{0}, v_{0}\right) \in\left(C^{\nu}(\bar{\Omega})\right)^{2}$ such that $u_{0}>0, v_{0} \geq 0$ and $\left.u_{0}\right|_{\partial \Omega}=\left.v_{0}\right|_{\partial \Omega}=0$, the solution of (4.1) converges to $\left(\bar{\theta}_{\lambda}, 0\right)$, as $t \rightarrow \infty$, uniformly in $\bar{\Omega}$, i.e., $\left(\bar{\theta}_{\lambda}, 0\right)$ is a global attractor.

(ii) If

$$
\lambda<\sigma_{1}\left[d_{1}, q_{1}+B \bar{\theta}_{\mu}, \ell_{2}\right], \quad \mu>\sigma_{1}\left[d_{2}, q_{2}+C \bar{\theta}_{\lambda}, m_{2}\right],
$$

and (2.2) does not admit a coexistence state, then $\left(0, \bar{\theta}_{\mu}\right)$ is a global attractor.

The proof of this theorem can be accomplished arguing as in [11], pages 856-857. In case (i), $v$ has upper solutions arbitrarily close to 0 as $t \rightarrow \infty$ and the global attractive character of $\bar{\theta}_{\lambda}$ for the logistic problem completes the proof. By symmetry, part (ii) follows. In the last paragraph of [11], page 858, it was claimed that the same technique provides us with Theorem 4.1. This is not right, because the construction of lower solutions is also required to prove Theorem 4.1. From Theorems 3.10 and 4.4 we obtain the following result, which is a substantial improvement of the extinction theorems of [5] and [14]. In both references it was assumed that $(\lambda, \mu) \in E_{\lambda}^{0} \cup E_{\mu}^{0}$.

Corollary 4.5. (i) If $(\lambda, \mu) \in \bigcup_{n=0}^{\infty} E_{\lambda}^{n}$ then for every pair of initial data $\left(u_{0}, v_{0}\right)$ $\in C^{\nu}(\bar{\Omega}) \times C^{\nu}(\bar{\Omega})$, such that $u_{0}>0, v_{0} \geq 0$ and $u_{0}=v_{0}=0$ on $\partial \Omega$, the solution $\left(u\left(x, t, u_{0}, v_{0}\right), v\left(x, t, u_{0}, v_{0}\right)\right)$ of $(4.1)$ converges to $\left(\bar{\theta}_{\lambda}, 0\right)$, as $t \rightarrow \infty$, uniformly in $\bar{\Omega}$, i.e., $\left(\bar{\theta}_{\lambda}, 0\right)$ is a global attractor.

(ii) If $(\lambda, \mu) \in \bigcup_{n=0}^{\infty} E_{\mu}^{n}$ then $\left(0, \bar{\theta}_{\mu}\right)$ is a global attractor.

\section{FIXED POINT INDEX: MULTIPLICITY RESULTS}

Consider the family of problems

$$
\begin{aligned}
& -d_{1} \Delta u+q_{1}(x) u=\lambda \ell_{2}(x) u-A(x) u^{2}-\gamma B(x) u v, \\
& -d_{2} \Delta v+q_{2}(x) v=\mu m_{2}(x) v-D(x) v^{2}-\gamma C(x) u v,
\end{aligned} \quad u, v \in U,
$$

where $\gamma \in[0,1]$. Observe that there are a priori bounds uniformly in $\gamma$ for the componentwise nonnegative solutions of (5.1). In particular, there exist two bounded open sets in $U$, say $N_{1}$ and $N_{2}$, such that if $(u, v) \in P_{U} \times P_{U}$ is a pair solving $(5.1)$ for some $\gamma \in[0,1]$ then $(u, v) \in N \equiv N_{1} \times N_{2}$. Let $M>0$ be large enough so that $(M+\lambda) \ell_{2}-A u-\gamma B v>0$ and $(M+\mu) m_{2}-$ $\gamma C u-D v>0$, for every $(\gamma, u, v) \in[0,1] \times N$, and $\sigma_{1}\left[d_{1}, q_{1}+M \ell_{2}, \ell_{2}\right]>0$, 
$\sigma_{1}\left[d_{2}, q_{2}+M m_{2}, m_{2}\right]>0$. Consider the operator $H:[0,1] \times N \rightarrow U^{2}$ defined by

$$
H(\gamma, u, v)=\left(\begin{array}{c}
K_{1}\left[(M+\lambda) \ell_{2}-A u-\gamma B v\right] u \\
K_{2}\left[(M+\mu) m_{2}-\gamma C u-D v\right] v
\end{array}\right),
$$

where $K_{1} \equiv\left[-d_{1} \Delta+q_{1}+M \ell_{2}\right]^{-1}$ and $K_{2} \equiv\left[-d_{2} \Delta+q_{2}+M m_{2}\right]^{-1} \cdot H(\gamma, \cdot, \cdot)$ is a compact positive differentiable operator whose fixed points are the componentwise nonnegative solutions of (5.1). As $N$ contains all its fixed points, the fixed point index of $H(\gamma, \cdot, \cdot)$ over $N$ with respect to the cone $P_{U} \times P_{U}$ makes sense. The main result of this section is the following.

Theorem 5.1. Assume (3.9), i.e., $\lambda>\sigma_{1,1}, \mu>\sigma_{1,2}$. Then the following holds:

(i) $i_{P_{U} \times P_{U}}(H(1, \cdot, \cdot), N)=1$;

(ii) $(0,0)$ is an isolated solution of $H(1, \cdot, \cdot)$ such that

$$
i_{P_{U} \times P_{U}}(H(1, \cdot, \cdot),(0,0))=0 ;
$$

(iii) $i_{P_{U} \times P_{U^{\prime}}}\left(H(1, \cdot, \cdot),\left(0, \bar{\theta}_{\mu}\right)\right)=0$ if $\lambda>\sigma_{1}\left[d_{1}, q_{1}+B \bar{\theta}_{\mu}, \ell_{2}\right]$;

(iv) $i_{P_{U} \times P_{U}}\left(H(1, \cdot, \cdot),\left(0, \bar{\theta}_{\mu}\right)\right)=1$ if $\lambda<\sigma_{1}\left[d_{1}, q_{1}+B \bar{\theta}_{\mu}, \ell_{2}\right]$;

(v) $i_{P_{U^{\prime}} \times P_{U}}\left(H(1, \cdot, \cdot),\left(\bar{\theta}_{\lambda}, 0\right)\right)=0$ if $\mu>\sigma_{1}\left[d_{2}, q_{2}+C \bar{\theta}_{\lambda}, m_{2}\right]$;

(vi) $i_{P_{U} \times P_{U}}\left(H(1, \cdot, \cdot),\left(\bar{\theta}_{\lambda}, 0\right)\right)=1$ if $\mu<\sigma_{1}\left[d_{2}, q_{2}+C \bar{\theta}_{\lambda}, m_{2}\right]$.

Proof. To prove part (i) we shall use Lemma 13.1 of [1]. From the homotopy invariance property we find that $i_{P_{U} \times P_{U}}(H(1, \cdot, \cdot), N)=i_{P_{U} \times P_{U}}(H(0, \cdot, \cdot), N)$ $=\prod_{j=1}^{2} i_{P_{U}}\left(H_{j}, N_{j}\right)$, where $H_{1}(u)=K_{1}\left[(M+\lambda) \ell_{2}-A u\right] u$ and $H_{2}(v)=$ $K_{2}\left[(M+\mu) m_{2}-D v\right] v$. Thus, to show (i) it is enough to show that

$$
i_{P_{U}}\left(H_{1}, N_{1}\right)=i_{P_{U}}\left(H_{2}, N_{2}\right)=1 \text {. }
$$

To prove (5.2) take $M$ larger, if necessary, so that $M+\sigma_{1,1}-1>0, M+$ $\sigma_{1,2}-1>0$ and consider the homotopies

$$
\begin{gathered}
G_{1}(\gamma, u)=K_{1}\left[\left(M+\sigma_{1,1}-1\right) \ell_{2}+\gamma\left(\left(\lambda-\sigma_{1,1}+1\right) \ell_{2}-A u\right)\right] u, \\
G_{2}(\gamma, v)=K_{2}\left[\left(M+\sigma_{1,2}-1\right) m_{2}+\gamma\left(\left(\mu-\sigma_{1,2}+1\right) m_{2}-D v\right)\right] v .
\end{gathered}
$$

It follows by homotopy invariance that

$$
i_{P_{U}}\left(H_{j}, N_{j}\right)=i_{P_{U}}\left(G_{j}(1, \cdot), N_{j}\right)=i_{P_{U}}\left(G_{j}(0, \cdot), N_{j}\right)=i_{P_{U}}\left(G_{j}(0, \cdot), 0\right),
$$

for $j=1,2$. Moreover, $G_{1}(0, \cdot)$ and $G_{2}(0, \cdot)$ have spectral radius

$$
\operatorname{spr} G_{1}(0, \cdot)=\frac{M+\sigma_{1,1}-1}{M+\sigma_{1,1}}<1, \quad \operatorname{spr} G_{2}(0, \cdot)=\frac{M+\sigma_{1,2}-1}{M+\sigma_{1,2}}<1,
$$

and hence (5.2) follows as an application of Lemma 13.1 of [1]. This completes the proof of part (i).

We now prove (ii). By differentiating $H(1, \cdot, \cdot)$ with respect to $(u, v)$ at $(0,0)$, we find that

$$
D_{(u, v)} H(1,0,0)(u, v)=\left(\begin{array}{c}
K_{1}(M+\lambda) \ell_{2} u \\
K_{2}(M+\mu) m_{2} v
\end{array}\right) .
$$

As $\lambda>\sigma_{1,1}$ and $\mu>\sigma_{1,2}$, the operator $I-D_{(u, v)} H(1,0,0)$ is invertible on $P_{U}^{2}$, i.e., one is not an eigenvalue to a positive eigenvector of $D_{(u, v)} H(1,0,0)$. Moreover,

$$
D_{(u, v)} H(1,0,0)\left(\varphi_{1}\left[d_{1}, q_{1}, \ell_{2}\right], 0\right)=\frac{M+\lambda}{M+\sigma_{1,1}} \cdot\left(\varphi_{1}\left[d_{1}, q_{1}, \ell_{2}\right], 0\right),
$$


and hence $D_{(u, v)} H(1,0,0)$ has a positive eigenfunction to an eigenvalue greater than one. Once again, Lemma 13.1 of [1] completes the proof.

To compute the indices of the semitrivial coexistence states we shall use the abstract theory in [9] as was done in [22]. First we must collect some notations and results from [9] as applied to our situation here. We are working with the ordered Banach space $(X, W), X=U^{2}, W=P_{U}^{2}$, and we want to compute the indices of the semitrivial coexistence states $y=\left(\bar{\theta}_{\lambda}, 0\right)$ and $y=\left(0, \bar{\theta}_{\mu}\right)$. Thus, the sets introduced in [9] by

$$
W_{y} \equiv\{x \in X: y+\gamma x \in W \text { for some } \gamma>0\}
$$

and $S_{y} \equiv\left\{x \in \bar{W}_{y}:-x \in \bar{W}_{y}\right\}$ become $W_{\left(\bar{\theta}_{\lambda}, 0\right)}=U \times P_{U}, W_{\left(0, \bar{\theta}_{\mu}\right)}=$ $P_{U} \times U$, and $S_{\left(\bar{\theta}_{\lambda}, 0\right)}=U \times\{0\}, S_{\left(0, \bar{\theta}_{\mu}\right)}=\{0\} \times U$. In both cases, the spaces $S_{y}$ have complements, say $M_{y}$, in $X$. Concretely, $M_{\left(\bar{\theta}_{\lambda}, 0\right)}=\{0\} \times U$ and $M_{\left(0, \bar{\theta}_{\mu}\right)}=U \times\{0\}$. Now consider continuous projections $P_{\left(\bar{\theta}_{\lambda}, 0\right)}(u, v)=(0, v)$, $P_{\left(0, \bar{\theta}_{\mu}\right)}(u, v)=(u, 0)$. Then the following result holds, [9], [22].

Lemma 5.2. (i) If $I-D_{x} H(1, y)$ is an invertible operator on $X$ and the spectral radius of $\left.P_{y} D_{x} H(1, y)\right|_{M_{y}}$ is greater than one, then $i_{W}(H(1, \cdot), y)=0$.

(ii) If $I-D_{x} H(1, y)$ is an invertible operator on $X$ and $\operatorname{spr}\left(\left.P_{y} D_{x} H(1, y)\right|_{M_{y}}\right)$ $<1$, then $i_{W}(H(1, \cdot), y)=(-1)^{\chi}$, where $\chi$ is the sum of the multiplicities of the eigenvalues of $D_{x} H(1, y)$ greater than one.

(iii) If $I-D_{x} H(1, y)$ is invertible on $W_{y}$ instead of $X$ and there is some $w \in W_{y}$ such that the equation $\left(I-D_{x} H(1, y)\right) x=w$ has no solution $x \in W_{y}$, then $i_{W}(H(1, \cdot), y)=0$.

We now show (v). By symmetry, also follows (iii). Assume

$$
\mu>\sigma_{1}\left[d_{2}, q_{2}+C \bar{\theta}_{\lambda}, m_{2}\right] .
$$

By differentiating with respect to $(u, v)$, we obtain

$$
D_{(u, v)} H\left(1, \bar{\theta}_{\lambda}, 0\right)(u, v)=\left(\begin{array}{c}
K_{1}\left[\left((M+\lambda) \ell_{2}-2 A \bar{\theta}_{\lambda}\right) u-B \bar{\theta}_{\lambda} v\right] \\
K_{2}\left[(M+\mu) m_{2}-C \bar{\theta}_{\lambda}\right] v
\end{array}\right) .
$$

Because of the choice of $M$, the operator $D_{(u, v)} H\left(1, \bar{\theta}_{\lambda}, 0\right)$ maps $U \times P_{U}=$ $W_{\left(\bar{\theta}_{\lambda}, 0\right)}$ into $U \times P_{U}$. We claim that $I-D_{(u, v)} H\left(1, \bar{\theta}_{\lambda}, 0\right)$ is an invertible operator on $U \times P_{U}$. To show this, consider $(u, \varphi) \in U \times P_{U}$ such that

$$
K_{1}\left[\left((M+\lambda) \ell_{2}-2 A \bar{\theta}_{\lambda}\right) u-B \bar{\theta}_{\lambda} \varphi\right]=u, \quad K_{2}\left[(M+\mu) m_{2}-C \bar{\theta}_{\lambda}\right] \varphi=\varphi .
$$

The second equation of (5.3) can be written as $\left(-d_{2} \Delta+q_{2}+C \bar{\theta}_{\lambda}\right) \varphi=\mu m_{2} \varphi$. As $\mu>\sigma_{1}\left[d_{2}, q_{2}+C \bar{\theta}_{\lambda}, m_{2}\right]$, we find that $\varphi=0$ and so the first equation of (5.3) becomes $\left(-d_{1} \Delta+q_{1}+2 A \bar{\theta}_{\lambda}\right) u=\lambda \ell_{2} u$. Since $\bar{\theta}_{\lambda}$ does not degenerate, it follows that $u=0$ and the claim above follows. We now show that the operator $I-D_{(u, v)} H\left(1, \bar{\theta}_{\lambda}, 0\right): U \times P_{U} \rightarrow U \times P_{U}$ does not have full rank. Then, part (v) follows from Lemma 5.2(iii). To show this we argue by contradiction assuming that given $(\varphi, \psi) \in U \times P_{U}$ arbitrary the following system has a solution $(u, v) \in U \times P_{U}$.

$u-K_{1}\left[\left((M+\lambda) \ell_{2}-2 A \bar{\theta}_{\lambda}\right) u-B \bar{\theta}_{\lambda} v\right]=\varphi, \quad v-K_{2}\left[(M+\mu) m_{2}-C \bar{\theta}_{\lambda}\right] v=\psi$. 
In particular, for every $p \in P_{U}$ the solution of $v-K_{2}\left[(M+\mu) m_{2}-C \bar{\theta}_{\lambda}\right] v=K_{2} p$ is positive. The last equation can be written as

$$
\left(-d_{2} \Delta+q_{2}+C \bar{\theta}_{\lambda}-\mu m_{2}\right) v=p .
$$

Hence the proof of this part will be concluded if we show that equality (5.4) cannot be solved with $v \geq 0$ for each $p \geq 0$. If the inverse operator $\left(-d_{2} \Delta+q_{2}+C \bar{\theta}_{\lambda}-\mu m_{2}\right)^{-1}$ exists, then it should be order preserving, which contradicts the assumption $\mu>\sigma_{1}\left[d_{2}, q_{2}+C \bar{\theta}_{\lambda}, m_{2}\right]$. Thus, there exists $u \in U-\{0\}$ such that $\left(-d_{2} \Delta+q_{2}+C \bar{\theta}_{\lambda}-\mu m_{2}\right) u=0$. Let $p \in P_{U}$ be such that $\int_{\Omega} p u \neq 0$ and suppose that (5.4) has a solution $v \in P_{U}$. Then

$\int_{\Omega} p u=\int_{\Omega} u\left(-d_{2} \Delta+q_{2}+C \bar{\theta}_{\lambda}-\mu m_{2}\right) v=\int_{\Omega} v\left(-d_{2} \Delta+q_{2}+C \bar{\theta}_{\lambda}-\mu m_{2}\right) u=0$, which contradicts the choice of $p$. This shows parts (iii) and (v) of Theorem 5.1 .

We now show part (vi). Part (iv) follows by symmetry. Assume $\mu<$ $\sigma_{1}\left[d_{2}, q_{2}+C \bar{\theta}_{\lambda}, m_{2}\right]$. We claim that $I-D_{(u, v)} H\left(1, \bar{\theta}_{\lambda}, 0\right)$ is invertible on $U^{2}$. To show this claim, consider $u, v \in U$ such that

$$
K_{1}\left[\left((M+\lambda) \ell_{2}-2 A \bar{\theta}_{\lambda}\right) u-B \bar{\theta}_{\lambda} v\right]=u, \quad K_{2}\left[(M+\mu) m_{2}-C \bar{\theta}_{\lambda}\right] v=v .
$$

Then $\left(-d_{1} \Delta+q_{1}\right) u=\left(\lambda \ell_{2}-2 A \bar{\theta}_{\lambda}\right) u-B \bar{\theta}_{\lambda} v$ and $\left(-d_{2} \Delta+q_{2}\right) v=\left(\mu m_{2}-C \bar{\theta}_{\lambda}\right) v$. As $\mu<\sigma_{1}\left[d_{2}, q_{2}+C \bar{\theta}_{\lambda}, m_{2}\right], v=0$ and so $\left(-d_{1} \Delta+q_{1}\right) u=\left(\lambda \ell_{2}-2 A \bar{\theta}_{\lambda}\right) u$. Due to the fact that $\bar{\theta}_{\lambda}$ does not degenerate we find that $u=0$. This shows the claim above. As $M_{\left(\bar{\theta}_{\lambda}, 0\right)}=\{0\} \times U$, if we show that the operator

$$
P_{\left(\bar{\theta}_{\lambda}, 0\right)} D_{(u, v)} H\left(1, \bar{\theta}_{\lambda}, 0\right)(0, v)=K_{2}\left[(M+\mu) m_{2}-C \bar{\theta}_{\lambda}\right] v
$$

has spectral radius less than one, then it follows from Lemma 5.2(ii) that

$$
i_{P_{U} \times P_{U}}\left(H(1, \cdot, \cdot),\left(\bar{\theta}_{\lambda}, 0\right)\right)=(-1)^{\chi},
$$

where $\chi$ is the sum of multiplicities of the eigenvalues of $D_{(u, v)} H\left(1, \bar{\theta}_{\lambda}, 0\right)$ greater than one. The spectral radius of $K_{2}\left[(M+\mu) m_{2}-C \bar{\theta}_{\lambda}\right]$ is the unique real positive number, say $r$, such that $\sigma_{1}\left[d_{2}, q_{2}+\frac{1}{r} C \bar{\theta}_{\lambda}, m_{2}\right]=\frac{M+\mu}{r}-M$, which can be written as

$$
\sigma_{1}\left[d_{2}, q_{2}+\frac{M}{m_{2}}-\frac{1}{r}\left(\frac{M+\mu}{m_{2}}-C \bar{\theta}_{\lambda}\right), m_{2}\right]=0 .
$$

If necessary, choose $M$ larger so that $\frac{M+\mu}{m_{2}}-C \bar{\theta}_{\lambda}>0$. Define

$$
f(s)=\sigma_{1}\left[d_{2}, q_{2}+\frac{M}{m_{2}}-\frac{1}{s}\left(\frac{M+\mu}{m_{2}}-C \bar{\theta}_{\lambda}\right), m_{2}\right] .
$$

The mapping $s \rightarrow f(s)$ is strictly increasing. As $\mu<\sigma_{1}\left[d_{2}, q_{2}+C \bar{\theta}_{\lambda}, m_{2}\right]$, $f(1)>0$ and hence $r<1$ and (5.5) follows. We claim that $\chi=0$. To show this, let $\varepsilon$ be an arbitrary eigenvalue of $D_{(u, v)} H\left(1, \bar{\theta}_{\lambda}, 0\right)$ with corresponding eigenfunction $(u, v)$. Then

$$
K_{1}\left[\left((M+\lambda) \ell_{2}-2 A \bar{\theta}_{\lambda}\right) u-B \bar{\theta}_{\lambda} v\right]=\varepsilon u, \quad K_{2}\left[(M+\mu) m_{2}-C \bar{\theta}_{\lambda}\right] v=\varepsilon v .
$$

and we distinguish two cases. If $v \neq 0$, then $\varepsilon$ is an eigenvalue of $K_{2}\left[(M+\mu) m_{2}-C \bar{\theta}_{\lambda}\right]$ and since the spectral radius of this operator is less than 
one, $\varepsilon$ is less than one also. If $v=0$, then $u \neq 0$ and $K_{1}\left[(M+\lambda) \ell_{2}-2 A \bar{\theta}_{\lambda}\right] u=$ $\varepsilon u$. Thus, $\varepsilon \leq \operatorname{spr} K_{1}\left[(M+\lambda) \ell_{2}-2 A \bar{\theta}_{\lambda}\right]<1$, because $\sigma_{1}\left[d_{1}, q_{1}+2 A \bar{\theta}_{\lambda}, \ell_{2}\right]>$ $\sigma_{1}\left[d_{1}, q_{1}+A \bar{\theta}_{\lambda}, \ell_{2}\right]=\lambda$. Therefore, $\chi=0$. This completes the proof.

From Theorem 5.1 we can obtain the existence result of Theorem 3.1(c) and the following result which complements Theorem 3.1(c).

Theorem 5.3. Assume

$$
\lambda<\sigma_{1}\left[d_{1}, q_{1}+B \bar{\theta}_{\mu}, \ell_{2}\right], \quad \mu<\sigma_{1}\left[d_{2}, q_{2}+C \bar{\theta}_{\lambda}, m_{2}\right] .
$$

Then (2.2) has a coexistence state. Moreover, the sum of the indices of the coexistence states of (2.2) is -1 .

Proof. Parts (i), (ii), (iv) and (vi) of Theorem 5.1 complete the proof.

Remark 5.4. Assume that all the coexistence states of (2.2) are nondegenerate. Then, it follows from Theorem 11.4 of [1] that the index of each coexistence state $\left(u_{0}, v_{0}\right)$ of $(2.2)$ is $(-1)^{m}$, where $m$ is the sum of the multiplicities of all the eigenvalues of $D_{(u, v)} H\left(1, u_{0}, v_{0}\right)$ which are outside of the unit disk. Since we are assuming that all the coexistence states are nondegenerate, they are isolated because they are fixed points of $H(1, \cdot, \cdot)$ which is compact. Thus, (2.2) has a finite number of coexistence states. If (5.6) is satisfied then the sum of the indices of the coexistence states is -1 and hence (2.2) has an odd number of coexistence states $2 k+1$, for some $k \geq 0 ; k+1$ of them are unstable and the remaining $k$ have index 1 . Similarly, if we assume (3.2) then the sum of the indices of the coexistence states of (2.2) is +1 and hence (2.2) also has an odd number of coexistence states $2 k+1$, for some $k \geq 0$. Now, $k$ of them are unstable and the remaining $k+1$ have index 1 . Theorem 3.1(c) guarantees that at least one of them is asymptotically stable.

Remark 5.5. Assume that $\{x \in \Omega: b(x)=0\}$ and $\{x \in \Omega: c(x)=0\}$ have Lebesgue measure zero. Then, it follows from Remark 3.13 that (5.6) holds provided $\|B\|_{\infty}$ and $\|C\|_{\infty}$ are large enough.

Remark 5.6. Under condition (5.6) one does not always expect uniqueness, even for the simplest model (3.4). Consider (3.4) with $a=d=1, \lambda=\mu, b=c$ and assume that $\Omega$ is a ball. Then a second radially symmetric coexistence state (and also nonradially symmetric ones) emanates from the coexistence state $(u, v)$ with $u=v$ as we vary $b$ (cf. bottom of page 849 in [11]).

Remark 5.7. An analysis of bifurcation directions from the semitrivial coexistence states shows that (3.2) and (5.6) together are not necessary for coexistence, [4], [11], [12]. Because of this, we have to assume in the statement of Theorem 4.4 that (2.2) does not admit a coexistence state. Observe that Theorem 4.4 is optimal.

\section{A MAXIMUM PRINCIPLE}

Consider the following second order uniformly elliptic operators:

$$
L_{k}(D)=-\sum_{i, j=1}^{N} \alpha_{i j}^{k}(x) D_{i} D_{j}+\sum_{i=1}^{N} \alpha_{i}^{k}(x) D_{i}+\alpha_{0}^{k}(x), \quad k=1,2,
$$


where $\alpha_{i j}^{k}, \alpha_{i}^{k}, \alpha_{0}^{k} \in V$ for all $i, j \in\{1, \cdots, N\}$ and $k \in\{1,2\}$. Let $p_{1}$, $p_{2} \in V$ be such that $p_{i}(x)>0$ for every $x \in \Omega$ and $i=1,2$. In this section we study the linear elliptic system

$$
\left(\begin{array}{cc}
L_{1}(D) & 0 \\
0 & L_{2}(D)
\end{array}\right)\left(\begin{array}{l}
u \\
v
\end{array}\right)=\left(\begin{array}{cc}
0 & -p_{1}(x) \\
-p_{2}(x) & 0
\end{array}\right)\left(\begin{array}{l}
u \\
v
\end{array}\right)+\left(\begin{array}{l}
f \\
g
\end{array}\right), \quad u, v \in U,
$$

where $f, g \in V$. The Banach space $U^{2}$ will be considered as ordered by the cone $C_{U} \equiv P_{U} \times\left(-P_{U}\right)$. The interior of this cone, denoted by $\dot{C}_{U}$, is $\dot{C}_{U}=\dot{P}_{U} \times\left(-\dot{P}_{U}\right)$. Given $\left(u_{i}, v_{i}\right) \in U^{2}, i=1,2$, we shall write $\left(u_{1}, v_{1}\right) \geq$ $\left(u_{2}, v_{2}\right)$ if $\left(u_{1}-u_{2}, v_{1}-v_{2}\right) \in C_{U},\left(u_{1}, v_{1}\right)>\left(u_{2}, v_{2}\right)$ if $\left(u_{1}-u_{2}, v_{1}-v_{2}\right) \in$ $C_{U}-\{(0,0)\}$, and $\left(u_{1}, v_{1}\right) \gg\left(u_{2}, v_{2}\right)$ if $\left(u_{1}-u_{2}, v_{1}-v_{2}\right) \in \dot{C}_{U}$. Similarly, the Banach space $V$ is considered as ordered by the cone $C_{V}=P_{V} \times\left(-P_{V}\right)$ and $\geq,>$, $\gg$ have the same meaning.

Definition 6.1. It is said that (6.1) satisfies the maximum principle if given $(f, g) \in C_{V}-\{(0,0)\}$ any solution $(u, v)$ of $(6.1)$ lies in $C_{U}$. If this is the case we shall say simply that (6.1) satisfies MP.

Definition 6.2. It is said that (6.1) satisfies the strong maximum principle if given $(f, g) \in C_{V}-\{(0,0)\}$ any solution $(u, v)$ of $(6.1)$ lies in $\dot{C}_{U}$. If this is the case we shall say simply that (6.1) satisfies SMP.

The following result characterizes whether (6.1) satisfies SMP or not.

Theorem 6.3. The following conditions are equivalent:

C1: There exists $\left(\psi_{1}, \psi_{2}\right) \in \dot{C}_{U}$ such that $L_{1}(D) \psi_{1}>-p_{1}(x) \psi_{2}$ and $L_{2}(D) \psi_{2}$ $<-p_{2}(x) \psi_{1}$.

C2: The operators $L_{1}(D), L_{2}(D)$ are coercive and

$$
\operatorname{spr}\left[L_{1}^{-1}\left(p_{1} L_{2}^{-1}\left(p_{2} \cdot\right)\right)\right]<1 .
$$

C3: (6.1) satisfies $\mathbf{S M P .}$

C4: (6.1) satisfies MP.

C5: The operator

$$
\mathscr{M} \equiv\left(\begin{array}{ll}
L_{1}(D) & p_{1}(x) \\
p_{2}(x) & L_{2}(D)
\end{array}\right): U^{2} \rightarrow V^{2}
$$

has some positive principal eigenvalue, say $\Lambda_{1}(\mathscr{M})$. By principal eigenvalue we mean an eigenvalue to a positive eigenfunction $\left(\varphi_{1}, \varphi_{2}\right) \in \dot{C}_{U}$.

Moreover, if some of the previous conditions is satisfied then the principal eigenvalue $\Lambda_{1}(\mathscr{M})$ is simple and there is no other eigenvalue of $\mathscr{M}$ with a positive eigenfunction. Furthermore, $\mathscr{M}^{-1}: V^{2} \rightarrow V^{2}$ is a compact strongly positive endomorphism of $V$ and for every $\left(q_{1}, q_{2}\right) \in C_{U}-\{(0,0)\}$ the equation

$$
\lambda\left(\begin{array}{l}
u_{1} \\
u_{2}
\end{array}\right)-\mathscr{M}^{-1}\left(\begin{array}{l}
u_{1} \\
u_{2}
\end{array}\right)=\left(\begin{array}{l}
q_{1} \\
q_{2}
\end{array}\right)
$$

has exactly one positive solution $\left(u_{1}, u_{2}\right) \in \dot{C}_{U}$ if $\lambda>\frac{1}{\Lambda_{1}(\mathscr{M})}$, and no positive solution for $\lambda \leq \frac{1}{\Lambda_{1}(\mathscr{M})}$. The equation

$$
\frac{1}{\Lambda_{1}(\mathscr{M})}\left(\begin{array}{l}
u_{1} \\
u_{2}
\end{array}\right)-\mathscr{M}^{-1}\left(\begin{array}{l}
u_{1} \\
u_{2}
\end{array}\right)=-\left(\begin{array}{l}
q_{1} \\
q_{2}
\end{array}\right)
$$

has no positive solution. 
Proof. To prove this result we use the following result, which is Theorem 3.2 of [1].

Theorem 6.4. Let $(B, P)$ be an ordered Banach space such that $\dot{P}$ is nonempty. Let $T$ be a strongly positive compact endomorphism of $B$. Then the following is true:

(i) The spectral radius $\operatorname{spr} T$ is positive;

(ii) $\operatorname{spr} T$ is a simple eigenvalue of $T$ having a positive eigenvector and there is no other eigenvalue with a positive eigenvector;

(iii) $\operatorname{spr} T$ is a simple eigenvalue of $T^{*}$ having a positive eigenvector;

(iv) For every $y \in P-\{0\}$, the equation $\lambda x-T x=y$ has exactly one positive solution if $\lambda>\operatorname{spr} T$, and no positive solution for $\lambda \leq \operatorname{spr} T$. The equation $\operatorname{spr} T x-T x=-y$ has no positive solution.

We shall divide the proof in several steps.

C1 implies C2: Let $\psi_{1} \in \dot{P}_{U}, \psi_{2} \in-\dot{P}_{U}$ be such that $L_{1}(D) \psi_{1}>-p_{1}(x) \psi_{2}$ and $L_{2}(D) \psi_{2}<-p_{2}(x) \psi_{1}$. As $\psi_{2} \ll 0,-p_{1} \psi_{2}>0$ and so $L_{1} \psi_{1}>0$. Thus, it follows from the maximum principle for the case of one equation that $L_{1}(D)$ is coercive. Similarly, it follows from $L_{2}\left(-\psi_{2}\right)>p_{2} \psi_{1}>0$ and $-\psi_{2} \gg 0$ that $L_{2}(D)$ is coercive. Define $f \equiv L_{1} \psi_{1}+p_{1} \psi_{2}$ and $g \equiv L_{2} \psi_{2}+p_{2} \psi_{1}$. The assumptions imply $f>0$ and $g<0$. On the other hand, $\psi_{1}=-L_{1}^{-1}\left(p_{1} \psi_{2}\right)+$ $L_{1}^{-1} f, \psi_{2}=-L_{2}^{-1}\left(p_{2} \psi_{1}\right)+L_{2}^{-1} g$ and hence

$$
\psi_{1}-L_{1}^{-1}\left(p_{1} L_{2}^{-1}\left(p_{2} \psi_{1}\right)\right)=L_{1}^{-1} f-L_{1}^{-1}\left(p_{1} L_{2}^{-1} g\right)>0 \text {. }
$$

Finally, it follows from Theorem 6.4 that spr $\left[L_{1}^{-1}\left(p_{1} L_{2}^{-1}\left(p_{2} \cdot\right)\right)\right]<1$.

C2 implies C3: Pick $(f, g) \in C_{U}-\{(0,0)\}$ and consider (6.1). Then

$$
u-L_{1}^{-1}\left(p_{1} L_{2}^{-1}\left(p_{2} u\right)\right)=L_{1}^{-1} f-L_{1}^{-1}\left(p_{1} L_{2}^{-1} g\right)>0
$$

and since $\operatorname{spr}\left[L_{1}^{-1}\left(p_{1} L_{2}^{-1}\left(p_{2} \cdot\right)\right)\right]<1$ Theorem 6.4 implies $u>0$. Moreover, $v=-L_{2}^{-1}\left(p_{2} u\right)+L_{2}^{-1} g \ll 0$ and $u=-L_{1}^{-1}\left(p_{1} v\right)+L_{1}^{-1} f \gg 0$, which completes the proof. Obviously, $\mathrm{C} 3$ implies $\mathrm{C} 4$.

C3 implies C5: If (6.1) satisfies SMP then the operator $\mathscr{M}^{-1}: V^{2} \rightarrow V^{2}$ is compact and strongly order preserving. Thus, C3 implies C5 as well. The last claims of Theorem 6.3 follow easily from Theorem 6.4 applied to $\mathscr{M}^{-1}$. As for the case of one single equation $\Lambda_{1}(\mathscr{M})=\frac{1}{\operatorname{spr} \mathscr{M}^{-1}}$.

C5 implies C1: Let $\Lambda_{1}>0,\left(\psi_{1}, \psi_{2}\right) \in \dot{C}_{U}$ be such that $\mathscr{M}\left(\psi_{1}, \psi_{2}\right)=$ $\lambda_{1}\left(\psi_{1}, \psi_{2}\right)$. Then $L_{1} \psi_{1}=-p_{1} \psi_{2}+\lambda_{1} \psi_{1}>-p_{1} \psi_{2}$ and $L_{2} \psi_{2}=-p_{2} \psi_{1}+\lambda_{1} \psi_{2}<$ $-p_{1} \psi_{2}$, which completes the proof.

C4 implies C1: Assume that (6.1) satisfies MP. Then $\mathscr{M}\left(u_{1}, u_{2}\right)=0$ only has the solution $\left(u_{1}, u_{2}\right)=(0,0)$, because if this were not the case we would contradict MP. As $\mathscr{M}: U^{2} \rightarrow V^{2}$ is Fredholm with index zero, it follows from Banach's open mapping theorem that $\mathscr{M}$ is an isomorphism. Pick $\left(q_{1}, q_{2}\right) \in$ $\dot{C}_{U}$ and consider $\left(\psi_{1}, \psi_{2}\right)=\mathscr{M}^{-1}\left(q_{1}, q_{2}\right)$. As $(6.1)$ satisfies MP, $\left(\psi_{1}, \psi_{2}\right) \in$ $C_{U}-\{(0,0)\}$. Let $\lambda>0$ be large enough so that $L_{1}(D)+\lambda$ and $L_{2}(D)+\lambda$ satisfy the strong maximum principle. Then $\left(L_{1}(D)+\lambda\right) \psi_{1}=-p_{1} \psi_{2}+q_{1}+\lambda \psi_{1}$, 
$\left(L_{2}(D)+\lambda\right) \psi_{2}=-p_{2} \psi_{1}+q_{2}+\lambda \psi_{2}$ and hence $\psi_{1}=\left(L_{1}+\lambda\right)^{-1}\left(-p_{1} \psi_{2}+q_{1}+\lambda \psi_{1}\right) \gg$ 0 and $\psi_{2}=\left(L_{2}+\lambda\right)^{-1}\left(-p_{2} \psi_{1}+q_{2}+\lambda \psi_{2}\right) \ll 0$, which completes the proof.

Theorem 6.5. The boundary value problem

$$
\begin{array}{r}
\left(\begin{array}{cc}
L_{1}(D) & 0 \\
0 & L_{2}(D)
\end{array}\right)\left(\begin{array}{l}
\varphi \\
\psi
\end{array}\right)=\left(\begin{array}{cc}
0 & -p_{1}(x) \\
-p_{2}(x) & 0
\end{array}\right)\left(\begin{array}{l}
\varphi \\
\psi
\end{array}\right)+\Lambda\left(\begin{array}{c}
\varphi \\
\psi
\end{array}\right), \\
\varphi, \psi \in U,
\end{array}
$$

has a unique eigenvalue, denoted by $\Lambda_{1}$, to a positive eigenfunction $(\varphi, \psi) \in$ $C_{U}-\{(0,0)\}$. Moreover, $\Lambda_{1} \in \mathbb{R}$ and it is simple. Furthermore, $(\varphi, \psi) \in \dot{C}_{U}$, i.e., $\varphi \gg 0$ and $\psi \ll 0$, and whenever $\Lambda_{1}>0$ any other eigenvalue of (6.2) satisfies $|\Lambda|>\Lambda_{1}$. In particular, $\Lambda=0$ is not an eigenvalue.

Proof. Let $\left(\sigma_{1}\left(L_{1}\right), \varphi\right),\left(\sigma_{1}\left(L_{2}\right),-\psi\right)$ be the principal eigenpairs of $L_{1}$ and $L_{2}$, respectively. Let $\lambda$ be large enough so that $\left(\sigma_{1}\left(L_{1}\right)+\lambda\right) \varphi>-p_{1} \psi$ and $\left(\sigma_{1}\left(L_{2}\right)+\lambda\right) \psi<-p_{2} \varphi$. Then $(\varphi, \psi) \in \dot{C}_{U},\left(L_{1}+\lambda\right) \varphi>-p_{1} \psi$ and $\left(L_{2}+\lambda\right) \psi<-p_{2} \varphi$. Thus, it follows from Theorem 6.3 that $(\mathscr{M}+\lambda I)^{-1}$ : $V^{2} \rightarrow V^{2}$ is compact and strongly order preserving. The remaining claims follow from Theorem 6.4.

Remark 6.6. Because of the lack of symmetry, the eigenvalue problem (6.2) may exhibit complex eigenvalues.

\section{ChaRACTERIZING THE STABILITY}

In this section we use the maximum principle of Section 6 to characterize the asymptotic stability of a coexistence state $\left(u_{0}, v_{0}\right)$ of $(2.2)$ by means of the operator $\mathscr{K} \equiv K\left[u_{0}, v_{0}\right]$ defined in (4.11). It is well known that the stability of $\left(u_{0}, v_{0}\right)$ is given by $\operatorname{spr} D_{(u, v)} \mathscr{H}\left(u_{0}, v_{0}\right)$, where $\mathscr{H} \equiv H(1, \cdot, \cdot)$ is the operator introduced in Section 5 . Namely, $\left(u_{0}, v_{0}\right)$ is asymptotically stable if $\operatorname{spr} D_{(u, v)} \mathscr{H}\left(u_{0}, v_{0}\right)<1$ and unstable if $\operatorname{spr} D_{(u, v)} \mathscr{H}\left(u_{0}, v_{0}\right)>1$. The main result of this section is the following.

Theorem 7.1. Let $\left(u_{0}, v_{0}\right)$ be a coexistence state of (2.2) and consider $\mathscr{K} \equiv$ $K\left[u_{0}, v_{0}\right]$, where $K\left[u_{0}, v_{0}\right]$ is given by (4.11). Then, $\operatorname{spr} D_{(u, v)} \mathscr{H}\left(u_{0}, v_{0}\right)<1$ if, and only if, $\operatorname{spr} \mathscr{K}<1$ and $\operatorname{spr} D_{(u, v)} \mathscr{H}\left(u_{0}, v_{0}\right)>1$ if, and only if, $\operatorname{spr} \mathscr{K}>$ 1. In particular,

$$
\operatorname{spr} D_{(u, v)} \mathscr{H}\left(u_{0}, v_{0}\right)=1
$$

if, and only if, $\operatorname{spr} \mathscr{K}=1$.

Proof. We first show that $\operatorname{spr} \mathscr{K}<1$ implies $\operatorname{spr} D_{(u, v)} \mathscr{H}\left(u_{0}, v_{0}\right)<1$. Assume $\operatorname{spr} \mathscr{K}<1$ and let $\varrho=\operatorname{spr} D_{(u, v)} \mathscr{H}\left(u_{0}, v_{0}\right)$ be. An easy calculation shows that

$$
D_{(u, v)} \mathscr{H}\left(u_{0}, v_{0}\right)(u, v)=\varrho(u, v)
$$

if, and only if,

$$
\begin{aligned}
& \mathscr{L}_{1} u=n_{1}(x)\left(1-\varrho^{-1}\right) u-\varrho^{-1} B(x) u_{0} v, \\
& \mathscr{L}_{2} v=n_{2}(x)\left(1-\varrho^{-1}\right) v-\varrho^{-1} C(x) v_{0} u,
\end{aligned}
$$

where $\mathscr{L}_{1} \equiv L_{1}\left[u_{0}, v_{0}\right], \mathscr{L}_{2} \equiv L_{2}\left[u_{0}, v_{0}\right]$ are the operators defined in (4.10) and $n_{1}(x), n_{2}(x)$ are given by

$$
n_{1}=2 A u_{0}+B v_{0}-(\lambda+M) \ell_{2}, \quad n_{2}=2 D v_{0}+C u_{0}-(\mu+M) m_{2} .
$$


If necessary, choose $M$ larger so that $n_{1}<0$ and $n_{2}<0$ (see the definition of $\mathscr{H})$. As spr $\mathscr{K}<1$, arguing as in the proof of Theorem 4.2 it follows that $\left(u_{0}, v_{0}\right)$ is nondegenerate and hence $\varrho \neq 1$. We now show that $\varrho<1$. To prove this, we argue by contradiction assuming that $\varrho>1$. As $\mathscr{L}_{1}, \mathscr{L}_{2}$ are coercive, $\mathscr{K}=\mathscr{L}_{1}^{-1}\left(B u_{0} \mathscr{L}_{2}^{-1}\left(C v_{0} \cdot\right)\right)$ and $\operatorname{spr} \mathscr{K}<1$ it follows from Theorem 6.3 that there exist $\varphi \gg 0, \psi \ll 0$ and $\Lambda_{1}>0$ (unique) such that

$$
\mathscr{L}_{1} \varphi=-B u_{0} \psi+\Lambda_{1} \varphi, \quad \mathscr{L}_{2} \psi=-C v_{0} \varphi+\Lambda_{1} \psi .
$$

On the other hand, it follows from $\varrho>1, n_{1}<0$ and $n_{2}<0$ that

$$
\begin{aligned}
& -B u_{0} \psi \geq-\varrho^{-1} B u_{0} \psi>\left(1-\varrho^{-1}\right) n_{1} \varphi-\varrho^{-1} B u_{0} \psi, \\
& -C v_{0} \varphi \leq-\varrho^{-1} C v_{0} \varphi<\left(1-\varrho^{-1}\right) n_{2} \psi-\varrho^{-1} C v_{0} \varphi .
\end{aligned}
$$

From these inequalities and (7.2) we find that

$\left[\mathscr{L}_{1}-\Lambda_{1}-\left(1-\varrho^{-1}\right) n_{1}\right] \varphi>-\varrho^{-1} B u_{0} \psi, \quad\left[\mathscr{L}_{2}-\Lambda_{1}-\left(1-\varrho^{-1}\right) n_{2}\right] \psi<-\varrho^{-1} C v_{0} \varphi$ and hence Theorem 6.3 implies that there exist $\hat{\Lambda}_{1}>0$ (unique) and $\hat{\varphi} \gg 0$, $\hat{\psi} \ll 0$ (unique up to multiplicative constants) such that

$$
\begin{aligned}
& {\left[\mathscr{L}_{1}-\Lambda_{1}-\left(1-\varrho^{-1}\right) n_{1}\right] \hat{\varphi} }=-\varrho^{-1} B u_{0} \hat{\psi}+\hat{\Lambda}_{1} \hat{\varphi}, \\
& {\left[\mathscr{L}_{2}-\Lambda_{1}-\left(1-\varrho^{-1}\right) n_{2}\right] \hat{\psi}=-\varrho^{-1} C v_{0} \hat{\varphi}+\hat{\Lambda}_{1} \hat{\psi}, }
\end{aligned}
$$

which can be equivalently written as

$$
\begin{aligned}
& \mathscr{L}_{1} \hat{\varphi}=\left(1-\varrho^{-1}\right) n_{1} \hat{\varphi}-\varrho^{-1} B u_{0} \hat{\psi}+\left(\hat{\Lambda}_{1}+\Lambda_{1}\right) \hat{\varphi}, \\
& \mathscr{L}_{2} \hat{\psi}=\left(1-\varrho^{-1}\right) n_{2} \hat{\psi}-\varrho^{-1} C v_{0} \hat{\varphi}+\left(\hat{\Lambda}_{1}+\Lambda_{1}\right) \hat{\psi} .
\end{aligned}
$$

Moreover, Theorem 6.5 shows that any other eigenvalue $\tau$ of

$$
\begin{aligned}
& \mathscr{L}_{1} u=\left(1-\varrho^{-1}\right) n_{1} u-\varrho^{-1} B u_{0} v+\tau u, \quad u, v \in U, \\
& \mathscr{L}_{2} v=\left(1-\varrho^{-1}\right) n_{2} v-\varrho^{-1} C v_{0} u+\tau v, \quad u \quad
\end{aligned}
$$

satisfies $|\tau|>\hat{\Lambda}_{1}+\Lambda_{1}>0$ and it follows from the definition of $\varrho$ that $\tau=0$ is an eigenvalue of the previous boundary value problem. This contradiction shows that $\varrho<1$. The previous analysis also shows that $\varrho=1$ if, and only if, $\operatorname{spr} \mathscr{K}=1$.

We now show that $\operatorname{spr} \mathscr{K}>1$ implies $\operatorname{spr} D_{(u, v)} \mathscr{H}\left(u_{0}, v_{0}\right)>1$. Assume $\operatorname{spr} \mathscr{K}>1$. It follows from Theorem 6.3 that there exist $\Lambda_{1} \in \mathbb{R}$ (unique) and $\varphi \gg 0, \psi \ll 0$ (unique up to multiplicative constants) satisfying (7.2). If we show that $\Lambda_{1}<0$ the proof is completed. Indeed, if $\Lambda_{1}<0$ then we can argue as in the proof of Theorem 10.1 of [1] to show that $\left(u_{0}, v_{0}\right)$ is unstable and so $\operatorname{spr} D_{(u, v)} \mathscr{H}\left(u_{0}, v_{0}\right) \geq 1$. As $\operatorname{spr} \mathscr{H}>1$, necessarily $\operatorname{spr} D_{(u, v)} \mathscr{H}\left(u_{0}, v_{0}\right)>1$. To prove that $\Lambda_{1}<0$ we argue by contradiction assuming that $\Lambda_{1} \geq 0$. Then

$$
\mathscr{L}_{1} \varphi \geq-B u_{0} \psi>-\frac{1}{\sqrt{\operatorname{spr} \mathscr{K}}} B u_{0} \psi, \quad \mathscr{L}_{2} \psi \leq-C v_{0} \varphi<-\frac{1}{\sqrt{\operatorname{spr} \mathscr{K}}} C v_{0} \varphi
$$

and hence Theorem 6.3 shows the existence of $\hat{\Lambda}_{1}>0, \hat{\varphi} \gg 0$ and $\hat{\psi} \ll 0$ such that

$$
\mathscr{L}_{1} \hat{\varphi}=-\frac{1}{\sqrt{\operatorname{spr} \mathscr{K}}} B u_{0} \hat{\psi}+\hat{\Lambda}_{1} \hat{\varphi}, \quad \mathscr{L}_{2} \hat{\psi}=-\frac{1}{\sqrt{\operatorname{spr} \mathscr{K}}} C v_{0} \hat{\varphi}+\hat{\Lambda}_{1} \hat{\psi}
$$


An easy calculation shows that $\operatorname{spr} \mathscr{K} \hat{\varphi}>\mathscr{K} \hat{\varphi}$, which contradicts Theorem 6.4. Therefore, $\Lambda_{1}<0$. The proof is completed.

\section{Global continuation in $\beta$}

In this section we assume that $b(x)>0$ and $c(x)>0$ for every $x \in \Omega$ and consider $(3.19)_{\beta}$ regarding to $\beta \geq 0$ as a continuation parameter. We shall analyze the global qualitative behaviour of the branch of coexistence states $(u(\beta), v(\beta))$ constructed by continuation in $\beta$ from the unique coexistence state of $(3.19)_{0}$.

Theorem 8.1. Assume

$$
\lambda>\sigma_{1,1}, \quad \mu>\sigma_{1}\left[d_{2}, q_{2}+C \bar{\theta}_{\lambda}, m_{2}\right] .
$$

Let $\beta_{s}>0$ be such that $\lambda=\sigma_{1}\left[d_{1}, q_{1}+\beta_{s} B \bar{\theta}_{\mu}, \ell_{2}\right]$, whose existence and uniqueness is guaranteed by Remark 3.13 and denote by $(u(\beta), v(\beta)), \beta \geq 0$, the branch of nondegenerate coexistence states emanating from the unique coexistence state of $(3.19)_{0}$. Then some of the following options occur. Either (i) the branch has no bifurcation points and can be globally continued up to degeneracy at the semitrivial solution $\left(0, \bar{\theta}_{\mu}\right)$ at the value $\beta=\beta_{s}$, or (ii) the branch can be continued up to some value of $\beta$, say $\beta_{t}$, at which it turns backward, still being a regular branch (without bifurcation points).

In case (i) $(u(\beta), v(\beta))$ is asymptotically stable for $\beta \in\left[0, \beta_{s}\right)$, except at most for a finite number of $\beta$. In case (ii) the branch $(u(\beta), v(\beta))$ is asymptotically stable up to $\beta=\beta_{t}$, except at most for a finite number of $\beta$. Then it becomes unstable for some range $\left(\beta_{t}-\varepsilon, \beta_{t}\right), \varepsilon>0$, and hence $(3.19)_{\beta}$ has at least two coexistence states, one of them stable and the other unstable. Moreover, when $\beta_{t} \leq \beta_{s}$ there is $\varepsilon>0$ such that $(3.19)_{\beta}$ has at least three coexistence states for $\beta \in\left(\beta_{t}-\varepsilon, \beta_{t}\right)$.

In particular, no secondary bifurcation (nor Hopf bifurcation) occurs before a turning point arises.

Proof. Given $\beta \geq 0$ and a coexistence state $\left(u_{0}, v_{0}\right)$ of $(3.19)_{\beta}$ we define

$$
\mathscr{K}_{\left(\beta, u_{0}, v_{0}\right)}=L_{1}\left[\beta, u_{0}, v_{0}\right]^{-1}\left[\beta B u_{0} L_{2}\left[\beta, u_{0}, v_{0}\right]^{-1}\left(C v_{0} \cdot\right)\right] \text {, }
$$

where $L_{1}\left[\beta, u_{0}, v_{0}\right]$ and $L_{2}\left[\beta, u_{0}, v_{0}\right]$ are the operators introduced in (4.10) with $B(x)$ replaced by $\beta B(x)$. The operator $\mathscr{K}_{\left(\beta, u_{0}, v_{0}\right)}$ is compact and strongly order preserving. Arguing as in the proof of Theorem 4.2 we find that $(u(\beta)$, $v(\beta))$ is a regular curve as long as

$$
\operatorname{spr} \mathscr{K}_{(\beta, u(\beta), v(\beta))}<1 .
$$

For $\beta$ small enough (8.3) holds true. Moreover, it follows from Theorem 3.12 that $(3.19)_{\beta}$ does not admit a coexistence state if $\beta$ is large enough. Thus, there is $\beta_{c_{1}}>0$ such that $\operatorname{spr} \mathscr{K}_{(\beta, u(\beta), v(\beta))}<1$ if $\beta \in\left(0, \beta_{c_{1}}\right)$ and $\operatorname{spr} \mathscr{K}_{\left(\beta_{c_{1}}, u\left(\beta_{c_{1}}\right), v\left(\beta_{c_{1}}\right)\right)}=1$. On the other hand, differentiating $(3.19)_{\beta}$ with respect to $\beta$ we find that

(8.4)

$$
\mathscr{L}_{1}(\beta) u^{\prime}(\beta)=-\beta B u(\beta) v^{\prime}(\beta)-B u(\beta) v(\beta), \quad \mathscr{L}_{2}(\beta) v^{\prime}(\beta)=-C v(\beta) u^{\prime}(\beta),
$$

where ' stands for differentiation with respect to $\beta$,

$$
\mathscr{L}_{1}(\beta)=L_{1}[\beta, u(\beta), v(\beta)] \text { and } \mathscr{L}_{2}(\beta)=L_{2}[\beta, u(\beta), v(\beta)] \text {. }
$$




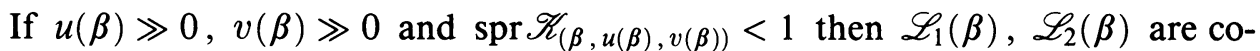
ercive and it follows from Theorem 6.3 that there exists a unique $\left(u^{\prime}(\beta), v^{\prime}(\beta)\right)$ solving (8.4). Moreover, $u^{\prime}(\beta) \ll 0$ and $v^{\prime}(\beta) \gg 0$. Thus, either $\beta_{c_{1}}=\beta_{s}$ and $\left(u\left(\beta_{c_{1}}\right), v\left(\beta_{c_{1}}\right)\right)=\left(0, \bar{\theta}_{\mu}\right)$, or $\left(u\left(\beta_{c_{1}}\right), v\left(\beta_{c_{1}}\right)\right)$ is a coexistence state. In any case, it follows from Theorem 7.1 that $(u(\beta), v(\beta))$ is asymptotically stable for $\beta \in\left[0, \beta_{c_{1}}\right)$. If the first alternative occurs then we are in case (i). If $\left(u\left(\beta_{c_{1}}\right), v\left(\beta_{c_{1}}\right)\right)$ is a coexistence state, we claim that $\left(\beta_{c_{1}}, u\left(\beta_{c_{1}}\right), v\left(\beta_{c_{1}}\right)\right)$ is a regular point of the curve $(\beta, u(\beta), v(\beta))$. To show this claim we argue as follows. The coexistence states of (2.2) are zeros of the operator $\mathscr{N}: \mathbb{R} \times U^{2} \rightarrow V^{2}$ defined by

$$
\mathscr{N}(\beta, u, v)=\left(\begin{array}{c}
\left(-d_{1} \Delta+q_{1}\right) u+A u^{2}+\beta B u v-\lambda \ell_{2} u \\
\left(-d_{2} \Delta+q_{2}\right) v+D v^{2}+C u v-\mu m_{2} v
\end{array}\right)
$$

In particular, $\mathcal{N}(\beta, u(\beta), v(\beta))=0$ if $\beta \in\left[0, \beta_{c_{1}}\right]$. A simple differentiation shows that

$$
D_{(u, v)} \mathscr{N}(\beta, u(\beta), v(\beta))=\left(\begin{array}{cc}
\mathscr{L}_{1}(\beta) & \beta B u(\beta) \\
C v(\beta) & \mathscr{L}_{2}(\beta)
\end{array}\right) .
$$

If $\beta \in\left[0, \beta_{c_{1}}\right)$ then inequality (8.3) holds and Theorem 6.3 implies that $D_{(u, v)} \mathcal{N}(\beta, u(\beta), v(\beta))$ is an isomorphism. As $\operatorname{spr} \mathscr{K}_{\left(\beta_{c_{1}}, u\left(\beta_{c_{1}}\right), v\left(\beta_{c_{1}}\right)\right)}=1$, $u\left(\beta_{c_{1}}\right) \gg 0$ and $v\left(\beta_{c_{1}}\right) \gg 0$, Theorem 6.3 implies that zero is a simple eigenvalue of $D_{(u, v)} \mathscr{N}\left(\beta_{c_{1}}, u\left(\beta_{c_{1}}\right), v\left(\beta_{c_{1}}\right)\right)$. In fact, $\beta_{c_{1}}$ is a value at which the maximum principle for the system (and so the stability of $(u(\beta), v(\beta))$ ) may be lost. To show the previous claim we only have to prove that

$$
\begin{aligned}
& D_{\beta} \mathcal{N}\left(\beta_{c_{1}}, u\left(\beta_{c_{1}}\right), v\left(\beta_{c_{1}}\right)\right) \\
& \quad=\left(\begin{array}{c}
B u\left(\beta_{c_{1}}\right) v\left(\beta_{c_{1}}\right) \\
0
\end{array}\right) \notin R\left[D_{(u, v)} \mathscr{N}\left(\beta_{c_{1}}, u\left(\beta_{c_{1}}\right), v\left(\beta_{c_{1}}\right)\right)\right],
\end{aligned}
$$

where $R[\cdot]$ stands for the range. To show this we argue by contradiction assuming that there is $(u, v) \in U^{2}$ such that $D_{(u, v)} \mathscr{N}\left(\beta_{c_{1}}, u\left(\beta_{c_{1}}\right), v\left(\beta_{c_{1}}\right)\right)(u, v)=$ $\left(B u\left(\beta_{c_{1}}\right) v\left(\beta_{c_{1}}\right), 0\right)$. Then, by eliminating $v$ from this system, we find that

$$
u=\mathscr{K}_{\left(\beta_{c_{1}}, u\left(\beta_{c_{1}}\right), v\left(\beta_{c_{1}}\right)\right)} u+\mathscr{L}_{1}^{-1}\left(\beta_{c_{1}}\right)\left(B u\left(\beta_{c_{1}}\right) v\left(\beta_{c_{1}}\right)\right) .
$$

Let $\varphi^{*} \gg 0$ be the unique positive function such that $\mathscr{K}_{\left(\beta_{c_{1}}, u\left(\beta_{c_{1}}\right), v\left(\beta_{c_{1}}\right)\right)}^{*} \varphi^{*}=\varphi^{*}$. Multiplying (8.6) by $\varphi^{*}$ and integrating on $\Omega$, it follows that

$$
\int_{\Omega} \varphi^{*} \mathscr{L}_{1}^{-1}\left(\beta_{c_{1}}\right)\left(B u\left(\beta_{c_{1}}\right) v\left(\beta_{c_{1}}\right)\right)=0
$$

which is impossible, because the integrand is positive. Thus, $\left(\beta_{c_{1}}, u\left(\beta_{c_{1}}\right), v\left(\beta_{c_{1}}\right)\right)$ is a regular point of the curve $(\beta, u(\beta), v(\beta))$, either a turning point, or an hysteresis point. Let $(\beta(p), \hat{u}(p), \hat{v}(p))$ be an analytic local parametrization of the curve of coexistence states at the simple fold point $\left(\beta_{c_{1}}, u\left(\beta_{c_{1}}\right), v\left(\beta_{c_{1}}\right)\right)$ by a certain real parameter $p \in(-\varepsilon, \varepsilon)$ such that $(\beta(0), \hat{u}(0), \hat{v}(0))=\left(\beta_{c_{1}}, u\left(\beta_{c_{1}}\right), v\left(\beta_{c_{1}}\right)\right)$ and $(\beta(p), \hat{u}(p), \hat{v}(p)), p \in$ $(-\varepsilon, 0)$, describes $(\beta, u(\beta), v(\beta))$ for $\beta<\beta_{c_{1}}, \beta \simeq \beta_{c_{1}}$. As the mapping $\beta \rightarrow(u(\beta), v(\beta))$ is analytic, the local parametrization $p \rightarrow(\beta(p), \hat{u}(p), \hat{v}(p))$ may be chosen analytic also. By construction, $\operatorname{spr} \mathscr{K}_{(\beta(p), \hat{u}(p), \hat{v}(p))}<1$ if $p<$ 0 and $\operatorname{spr} \mathscr{K}_{(\beta(0), \hat{u}(0), \hat{v}(0))}=1$. Moreover, either $\operatorname{spr} \mathscr{K}_{(\beta(p), \hat{u}(p), \hat{v}(p))}<1$ or 
$\operatorname{spr} \mathscr{K}_{(\beta(p), \hat{u}(p), \hat{v}(p))}>1$ for $p>0$ small enough, because the perturbation theory of [19] shows that $p \rightarrow \operatorname{spr} \mathscr{K}_{(\beta(p), \hat{u}(p), \hat{v}(p))}$ is analytic, excluding the case $\operatorname{spr} \mathscr{K}_{\left(\beta\left(p_{n}\right), \hat{u}\left(p_{n}\right), \hat{v}\left(p_{n}\right)\right)}=1$ for some sequence $p_{n}>0, p_{n} \rightarrow 0$, as $n \rightarrow \infty$. If $\operatorname{spr} \mathscr{K}_{(\beta(p), \hat{u}(p), \hat{v}(p))}<1, p>0$, then the branch can be continued to the right of $\beta_{c_{1}}$ up to the next value of $\beta$, say $\beta_{c_{2}}>\beta_{c_{1}}$ at which $\operatorname{spr} \mathscr{K}_{\left(\beta_{c_{2}}, u\left(\beta_{c_{2}}\right), v\left(\beta_{c_{2}}\right)\right)}=1$. In this case, $\left(\beta_{c_{1}}, u\left(\beta_{c_{1}}\right), v\left(\beta_{c_{1}}\right)\right)$ is a hysteresis point and it follows from Theorem 7.1 that $(u(\beta), v(\beta))$ is asymptotically stable for all $\beta \in\left(\beta_{c_{1}}, \beta_{c_{2}}\right)$. If $\operatorname{spr} \mathscr{K}_{(\beta(p), \hat{u}(p), \hat{v}(p))}>1, p>0$, then the branch turns backward and alternative (ii) occurs, i.e., $\left(\beta_{c_{1}}, u\left(\beta_{c_{1}}\right), v\left(\beta_{c_{1}}\right)\right)$ is a subcritical turning point and the branch becomes unstable after passing through it. The previous discussion may be repeated by arguing with $\beta_{c_{2}}$ as we did with $\beta_{c_{1}}$. In any case, the branch of coexistence states is a compact arc of analytic curve and so the set of points $\beta_{c_{n}}$ at which $\operatorname{spr} \mathscr{K}_{\left(\beta_{c_{n}}, u\left(\beta_{c_{n}}\right), v\left(\beta_{\left.c_{n}\right)}\right)\right.}=1$ is at most finite. After a finite number of steps either the branch degenerates at the semitrivial branch $\left(0, \bar{\theta}_{\mu}\right)$ or it has a turning point, because $u^{\prime}(\beta) \ll 0$ and $v^{\prime}(\beta) \gg 0$ as long as the branch is parametrized by $\beta$. The multiplicity result in case (ii) follows from Remark 5.4 .

Remark 8.2. Let $\mathscr{H}: \mathbb{R} \times N \rightarrow U^{2}$ be the operator defined by

$$
\mathscr{H}(\beta, u, v)=\left(\begin{array}{l}
K_{1}\left[(M+\lambda) \ell_{2}-A u-\beta B v\right] u \\
K_{2}\left[(M+\mu) m_{2}-C u-D v\right] v
\end{array}\right),
$$

where $K_{1}=\left[-d_{1} \Delta+q_{1}+M \ell_{2}\right]^{-1}, K_{2}=\left[-d_{2} \Delta+q_{2}+M m_{2}\right]^{-1}, M$ is a constant (large enough so that all the requirements in Section 5 hold) and $N$ is a bounded set of $U^{2}$ which contains any coexistence state of $(3.19)_{\beta}$ for $\beta \in\left[0, \beta_{e}\right)$, where $\beta_{e}$ is an extinction value (cf. Theorem 3.12). When alternative (ii) of Theorem 8.1 occurs the branch of coexistence states may be continued beyond the turning point as long as

$$
\operatorname{spr} D_{(u, v)} \mathscr{H}(\beta(p), \hat{u}(p), \hat{v}(p))>1, \quad 1 \notin \sigma\left[D_{(u, v)} \mathscr{C}(\beta(p), \hat{u}(p), \hat{v}(p))\right] .
$$

Moreover, $(\beta(p), \hat{u}(p), \hat{v}(p))$ is unstable for such range of $p$. Let $p_{0}$ be the first value of $p$ at which (8.7) fails. Two different situations may occur. Either (8.8)

$\operatorname{spr} D_{(u, v)} \mathscr{H}\left(\beta\left(p_{0}\right), \hat{u}\left(p_{0}\right), \hat{v}\left(p_{0}\right)\right)>1, \quad 1 \in \sigma\left[D_{(u, v)} \mathscr{H}\left(\beta\left(p_{0}\right), \hat{u}\left(p_{0}\right), \hat{v}\left(p_{0}\right)\right)\right]$, or $\operatorname{spr} D_{(u, v)} \mathscr{H}\left(\beta\left(p_{0}\right), \hat{u}\left(p_{0}\right), \hat{v}\left(p_{0}\right)\right)=1$. If (8.8) is satisfied then (generically) some new continuum of coexistence states bifurcates from the branch $(\beta(p), \hat{u}(p), \hat{v}(p))$ at $p=p_{0}$. If $\operatorname{spr} D_{(u, v)} \mathscr{H}\left(\beta\left(p_{0}\right), \hat{u}\left(p_{0}\right), \hat{v}\left(p_{0}\right)\right)=1$ then either $\left(\hat{u}\left(p_{0}\right), \hat{v}\left(p_{0}\right)\right)=\left(0, \bar{\theta}_{\mu}\right)$ or $\left(\hat{u}\left(p_{0}\right), \hat{v}\left(p_{0}\right)\right)$ is a coexistence state. If the last alternative occurs, we can argue as in the proof of Theorem 8.1 to show that $\left(\beta\left(p_{0}\right), \hat{u}\left(p_{0}\right), \hat{v}\left(p_{0}\right)\right)$ is a regular point of the curve $(\beta(p), \hat{u}(p), \hat{v}(p))$. Then, either the branch can be continued to the right of $p_{0}$, giving rise to a new arc of unstable coexistence states, or it has a supercritical turning point at $\left(\beta\left(p_{0}\right), \hat{u}\left(p_{0}\right), \hat{v}\left(p_{0}\right)\right)$ (with respect to $\beta$ ). If the last occurs, we obtain a new arc filled in by asymptotically stable coexistence states. This process may be repeated until condition (8.8). The analyticity of the spectral radius shows that in any case the number of turning points is finite.

The techniques above also show that under condition (8.1) any asymptotically stable coexistence state of $(3.19)_{\beta}$ lies in a global curve of coexistence states. 
Theorem 8.3. Assume (8.1). Let $\left(u_{0}, v_{0}\right)$ be a coexistence state of $(3.19)_{\beta}$ such that

$$
\operatorname{spr} D_{(u, v)} \mathscr{H}\left(\beta, u_{0}, v_{0}\right) \leq 1
$$

Then, there exists a maximal compact arc of analytic curve $(\beta(s), u(s), v(s))$, $s \in\left[s_{\alpha}, s_{\omega}\right], s_{\alpha}<0, s_{\omega}>0$, such that $(\beta(0), u(0), v(0))=\left(\beta, u_{0}, v_{0}\right)$ and the following conditions are satisfied:

(i) If $\beta\left(s_{\alpha}\right)=0$, then $\left(u\left(s_{\alpha}\right), v\left(s_{\alpha}\right)\right)$ is the unique coexistence state of $(3.19)_{0}$.

(ii) If $\beta\left(s_{\alpha}\right)>0$, then

$\operatorname{spr} D_{(u, v)} \mathscr{H}\left(\beta\left(s_{\alpha}\right), u\left(s_{\alpha}\right), v\left(s_{\alpha}\right)\right)>1, \quad 1 \in \sigma\left[D_{(u, v)} \mathscr{H}\left(\beta\left(s_{\alpha}\right), u\left(s_{\alpha}\right), v\left(s_{\alpha}\right)\right)\right]$.

(iii) Either $\beta\left(s_{\omega}\right)=\beta_{s}$ and $\left(u\left(s_{\omega}\right), v\left(s_{\omega}\right)\right)=\left(0, \bar{\theta}_{\mu}\right)$, or $\operatorname{spr} D_{(u, v)} \mathscr{H}\left(\beta\left(s_{\omega}\right), u\left(s_{\omega}\right), v\left(s_{\omega}\right)\right)>1, \quad 1 \in \sigma\left[D_{(u, v)} \mathscr{H}\left(\beta\left(s_{\omega}\right), u\left(s_{\omega}\right), v\left(s_{\omega}\right)\right)\right]$. In particular, $\left(u_{0}, v_{0}\right)$ is isolated. If in addition $\left(\beta, u_{0}, v_{0}\right)$ is not a turning point then either $\beta\left(s_{\alpha}\right)=0$ or the curve has a supercritical turning point (looking at the $(\beta, u)$-diagram) at some value $\beta\left(s_{t}\right)<\beta$. Similarly, either $\beta\left(s_{\omega}\right)=\beta_{s}$ or the curve has a subcritical turning point at some value $\beta\left(s_{t}\right)>\beta$.

Proof. From (8.9) and Theorem 7.1 we find that $\operatorname{spr} \mathscr{K}_{\left(\beta, u_{0}, v_{0}\right)} \leq 1$. Thus, arguing as in the proof of Theorem 8.1 it follows that $\left(\beta, u_{0}, v_{0}\right)$ is a regular zero of $\mathcal{N}$. In particular, $\left(u_{0}, v_{0}\right)$ is isolated and the local existence of the curve of coexistence states $(\beta(s), u(s), v(s))$ follows. The global behaviour follows arguing as in the proof of Theorem 8.1 and Remark 8.2.

\section{ChaRACTERIZING THE UNIQUenESS}

It follows from Theorems 3.1 and 5.3 that

$$
\left(\lambda-\sigma_{1}\left[d_{1}, q_{1}+\beta B \bar{\theta}_{\mu}, \ell_{2}\right]\right) \cdot\left(\mu-\sigma_{1}\left[d_{2}, q_{2}+C \bar{\theta}_{\lambda}, m_{2}\right]\right)>0
$$

is sufficient for the existence of a coexistence state of $(3.19)_{\beta}$. But an analysis of bifurcation directions from the semitrivial coexistence states shows that $(9.1)$ is not necessary for the existence of a coexistence state, [4], [11], [12]. Theorem 1 of [11] provides us with a deep understanding of the structure of the set of coexistence states of $(3.19)_{\beta}$ when (9.1) fails. The following result, which is a generalization of Theorem 1 in [11], will be used to characterize the supremum of the set of $\hat{\beta}$ such that $(3.19)_{\beta}$ has a unique coexistence state for all $\beta \in$ $[0, \hat{\beta})$. In this section we shall keep the notations introduced in Section 8 .

Theorem 9.1. Assume $\lambda>\sigma_{1,1}, \mu>\sigma_{1,2}$. Consider (3.19) $)_{\beta}$ with $C(x)$ replaced by $\gamma C(x)$, where $\gamma \geq 0$ is a real parameter. Let $\beta_{s}, \gamma_{s}$ be the unique values of $\beta$ and $\gamma$ such that $\lambda=\sigma_{1}\left[d_{1}, q_{1}+\beta_{s} B \bar{\theta}_{\mu}, \ell_{2}\right]$ and $\mu=$ $\sigma_{1}\left[d_{2}, q_{2}+\gamma_{s} C \bar{\theta}_{\lambda}, m_{2}\right]$. Let $\mathcal{I}$ denote the set of $(\beta, \gamma)$ such that $\beta>\beta_{s}$, $0 \leq \gamma<\gamma_{s}$ and $(3.19)_{\beta}$ has a coexistence state. Assume that $\mathscr{I}$ is nonempty. Then there exist $\beta_{1}>\beta_{s}, \gamma_{0} \in\left(0, \gamma_{s}\right)$, and a continuous strictly increasing function $f:\left[\beta_{s}, \beta_{1}\right] \rightarrow\left[\gamma_{0}, \gamma_{s}\right]$ such that $f\left(\beta_{s}\right)=\gamma_{0}, f\left(\beta_{1}\right)=\gamma_{s}$ and

$$
\mathscr{I}=\left\{(\beta, \gamma) \in\left(\beta_{s}, \beta_{1}\right] \times\left[\gamma_{0}, \gamma_{s}\right): f(\beta) \leq \gamma\right\} \text {. }
$$

Moreover, if $(\beta, \gamma) \in \dot{\mathcal{I}}$ (the interior of $\mathscr{I}$ ) then $(3.19)_{\beta}$ has at least two coexistence states. At least one of them is asymptotically stable with index 1. 
Furthermore, $(3.19)_{\beta}$ has an asymptotically stable coexistence state if $\beta=\beta_{s}$ and $\gamma \in\left(\gamma_{0}, \gamma_{s}\right)$, and a coexistence state if $\gamma=\gamma_{s}$ and $\beta \in\left(\beta_{s}, \beta_{1}\right)$.

Proof. As $\beta>\beta_{s}$ and $\gamma<\gamma_{s}$,

$$
\lambda<\sigma_{1}\left[d_{1}, q_{1}+\beta B \bar{\theta}_{\mu}, \ell_{2}\right] \text { and } \mu>\sigma_{1}\left[d_{2}, q_{2}+\gamma C \bar{\theta}_{\lambda}, m_{2}\right] .
$$

Thus, it follows from Theorem 5.1 that the sum of the indices of the coexistence states of $(3.19)_{\beta}$ is zero. The rest of the proof may be accomplished arguing as in [11], from the bottom of page 835 .

Remark 9.2. Assume that $\mathscr{I}$ is nonempty and consider $(\beta, \gamma) \in \dot{\mathcal{I}}$ such that all the coexistence states of $(3.19)_{\beta}$ are nondegenerate. As the sum of the indices of the coexistence states of $(3.19)_{\beta}$ is zero, it follows from Theorem 11.4 of [1] that $(3.19)_{\beta}$ has $2 k$ coexistence states for some $k \geq 1 ; k$ of them with index -1 and the other $k$ with index 1 . Those having index -1 are unstable. Due to Theorem 9.1, some coexistence state is asymtoptically stable (necessarily with index 1 ).

Remark 9.3. By symmetry, if $\mathcal{J}$ stands for the set of $(\gamma, \beta)$ such that $\gamma>\gamma_{s}$, $0 \leq \beta<\beta_{s}$ and $(3.19)_{\beta}$ has a coexistence state and we assume that $\mathcal{J}$ is nonempty then there exist $\gamma_{1}>\gamma_{s}, \beta_{0} \in\left(0, \beta_{s}\right)$, and a continuous strictly increasing function $g:\left[\gamma_{s}, \gamma_{1}\right] \rightarrow\left[\beta_{0}, \beta_{s}\right]$ such that $g\left(\gamma_{s}\right)=\beta_{0}, g\left(\gamma_{1}\right)=\beta_{s}$,

$$
\mathscr{J}=\left\{(\gamma, \beta) \in\left(\gamma_{s}, \gamma_{1}\right] \times\left[\beta_{0}, \beta_{s}\right): g(\gamma) \leq \beta\right\},
$$

and all the assertions of Theorem 9.1 hold.

The following result is a consequence from Theorems 3.1 and 9.1.

Corollary 9.4. Assume (8.1). Let $\mathscr{I}_{1}$ denote the set of $\beta \geq 0$ for which (3.19) $\beta$ has a coexistence state. Then, either $\mathscr{I}_{1}=\left[0, \beta_{s}\right)$ or $\mathscr{I}_{1}=\left[0, f^{-1}(1)\right]$, where $f$ is the function whose existence was shown by Theorem 9.1 (observe that $\left.f^{-1}(1) \geq \beta_{s}\right)$. In particular, $\mathscr{I}_{1}$ is an interval. Moreover, $(3.19)_{\beta}$ has an asymptotically stable coexistence state provided $\beta \in \dot{\mathscr{J}}_{1}$, the interior of $\mathscr{I}_{1}$. Furthermore, if $f^{-1}(1)>\beta_{s}$ then (3.19) ${ }_{\beta}$ has two coexistence states for each $\beta \in\left(\beta_{s}, f^{-1}(1)\right)$, at least.

From this result we can obtain the following substantial improvement of Corollary 4.3.

Theorem 9.5. Assume (8.1) and let $\beta_{g a}$ be the supremum of the set of $\hat{\beta}$ such that $(3.19)_{\beta}$ has a unique coexistence state for all $\beta \in[0, \hat{\beta})$. Then, either $\beta_{g a}=\beta_{s}$ or $\beta_{g a}<\beta_{s}$ and (3.19) $\beta_{\beta_{g}}$ has two coexistence states. In both cases $(u(\beta), v(\beta))$ is the unique coexistence state of (3.19) $\beta_{\beta}$ for all $\beta \in\left[0, \beta_{g a}\right)$. Moreover, $(u(\beta), v(\beta))$ is globally asymptotically stable and $\operatorname{spr} \mathscr{K}_{(\beta, u(\beta), v(\beta))} \leq 1$. Furthermore, if $\beta_{g a}<\beta_{s}$ then either (i) there exists a coexistence state $\left(u_{1}, v_{1}\right) \neq$ $\left(u\left(\beta_{g a}\right), v\left(\beta_{g a}\right)\right)$ such that $\operatorname{spr} \mathscr{K}_{\left(\beta_{g a}, u_{1}, v_{1}\right)}>1$, or (ii) $\operatorname{spr} \mathscr{K}_{\left(\beta_{g a}, u_{1}, v_{1}\right)}=1$ for any coexistence state $\left(u_{1}, v_{1}\right) \neq\left(u\left(\beta_{g a}\right), v\left(\beta_{g a}\right)\right)$ and given $\varepsilon>0$ small enough $(3.19)_{\beta_{g a}+\varepsilon}$ has at least three coexistence states; one of them, say $\left(u_{1}^{\varepsilon}, v_{1}^{\varepsilon}\right)$, satisfying $\operatorname{spr} \mathscr{K}_{\left(\beta_{g a}+\varepsilon, u_{1}^{\ell}, v_{1}^{e}\right)}>1$.

Proof. Corollary 4.3 implies $\beta_{g a}>0$. Moreover, it follows from Corollary 9.4 that $\beta_{g a} \leq \beta_{s}$. We claim that (3.19) $\beta_{\beta_{g a}}$ has two coexistence states whenever $\beta_{g a}<\beta_{s}$. To show this claim we argue by contradiction assuming that 
$\beta_{g a}<\beta_{s}$ and that $(3.19)_{\beta_{g a}}$ has a unique coexistence state. As $\beta_{g a}<\beta_{s}$, $\left(u\left(\beta_{g a}\right), v\left(\beta_{g a}\right)\right)$ is the unique coexistence state of $(3.19)_{\beta_{g a}}$. Moreover, from Theorem 8.1 and the definition of $\beta_{g a}$ it follows that the curve of coexistence states $(\beta, u(\beta), v(\beta))$ can be prolonged beyond $\beta_{g a}$. Otherwise the point $\left(\beta_{g a}, u\left(\beta_{g a}\right), v\left(\beta_{g a}\right)\right)$ would be a subcritical turning point contradicting the definition of $\beta_{g a}$. Let $\varepsilon>0$ be such that $(\beta, u(\beta), v(\beta))$ is defined for every $\beta \in\left[0, \beta_{g a}+\varepsilon\right]$. As this curve was obtained as an application of the Implicit Function Theorem, there exists an open subset $\mathscr{B} \subset \mathbb{R} \times U^{2}$ such that $(\beta, u(\beta), v(\beta)) \in \mathscr{B}$ for all $\beta \in\left[0, \beta_{g a}+\varepsilon\right]$ and any other coexistence state of $(3.19)_{\beta}, \beta \in\left[0, \beta_{g a}+\varepsilon\right]$, lies outside $\mathscr{B}$. As we are assuming that $\left(u\left(\beta_{g a}\right), v\left(\beta_{g a}\right)\right)$ is the unique coexistence state of $(3.19)_{\beta_{g a}}$, it follows from the definition of $\beta_{g a}$ that there exist two sequences, one of numbers $\varepsilon_{n}>0, n \geq 1$, $\lim _{n \rightarrow \infty} \varepsilon_{n}=0$, and the other of coexistence states, $\left(u_{n}, v_{n}\right), n \geq 1$, such that $\left(u_{n}, v_{n}\right)$ solves $(3.19)_{\beta_{g a}+\varepsilon_{n}}$ for every $n \geq 1$ and $\left(\beta_{g a}+\varepsilon_{n}, u_{n}, v_{n}\right) \notin \mathscr{B}$. Since uniform a priori bounds are available and $\mathscr{H}\left(\beta_{g a}+\varepsilon_{n}, u_{n}, v_{n}\right)=\left(u_{n}, v_{n}\right)$, $n \geq 1$, there exists a subsequence of $\left(u_{n}, v_{n}\right)$, again labelled by $n$, which converges to some solution couple $\left(u_{\infty}, v_{\infty}\right)$ of $(3.19)_{\beta_{g a}}$, as $n \rightarrow \infty$. As we are assuming that $\beta_{g a}<\beta_{s}$, necessarily $\left(u_{\infty}, v_{\infty}\right)$ is a coexistence state of (3.19) $\beta_{\beta_{g}}$. Moreover, $\left(\beta_{g a}, u_{\infty}, v_{\infty}\right) \notin \mathscr{B}$. This is a contradiction showing that $(3.19)_{\beta_{g a}}$ has at least two coexistence states. Observe that there is no ambiguity, because it may happen (3.19) ${\beta_{g a}+\varepsilon}$ has a unique coexistence state for $\varepsilon>0$ small. Those features show that $(3.19)_{\beta_{g a}}$ has two coexistence states (at least).

Assume that $\beta_{g a}<\beta_{s}$. If $\operatorname{spr} \mathscr{K}_{\left(\beta_{g a}, u_{1}, v_{1}\right)}<1$ for some coexistence state $\left(u_{1}, v_{1}\right) \neq\left(u\left(\beta_{g a}\right), v\left(\beta_{g a}\right)\right)$ then the Implicit Function Theorem shows that (3.19) $\beta_{\beta_{g}-\varepsilon}$ has at least two coexistence states for $\varepsilon>0$ small enough. Hence, $\operatorname{spr} \mathscr{K}_{\left(\beta_{g a}, u_{1}, v_{1}\right)} \geq 1$ for any coexistence state $\left(u_{1}, v_{1}\right) \neq\left(u\left(\beta_{g a}\right), v\left(\beta_{g a}\right)\right)$. Thus, either (i) is satisfied or $\operatorname{spr} \mathscr{K}_{\left(\beta_{g a}, u_{1}, v_{1}\right)}=1$ for any coexistence state $\left(u_{1}, v_{1}\right) \neq\left(u\left(\beta_{g a}\right), v\left(\beta_{g a}\right)\right)$. When the second alternative occurs, from the definition of $\beta_{g a}$ we find that all the points $\left(\beta_{g a}, u_{1}, v_{1}\right)$ provide us with supercritical turning points and hence the last assertion of the theorem follows (see the proof of Theorem 8.1). Notice that condition (8.1) eliminates the possibility of having a vertical segment of coexistence states joining the two semitrivial states. This completes the proof.

This result provides us with the following characterization.

Corollary 9.6. Assume (8.1) and consider $\beta_{0} \in\left(0, \beta_{s}\right]$ arbitrary. Then the following assertions are equivalent:

(i) The problem (3.19) $)_{\beta}$ has a unique coexistence state for every $\beta \in\left[0, \beta_{0}\right)$.

(ii) For every $\beta \in\left[0, \beta_{0}\right)$, any coexistence state $\left(u_{0}, v_{0}\right)$ of $(3.19)_{\beta}$ satisfies

$$
\operatorname{spr} \mathscr{K}_{\left(\beta, u_{0}, v_{0}\right)} \leq 1 .
$$

Condition (4.13) is the simplest sufficient condition for (9.2). It may be improved by replacing $\bar{\theta}_{\lambda}, \bar{\theta}_{\mu}, \underline{\theta}_{\lambda}$ and $\underline{\theta}_{\mu}$ by sharper upper and lower solutions. Modulo this sharpening, Corollary 9.6 tells us that (4.13) is the optimal condition for uniqueness. 


\section{ACKNOWLEDGMENTS}

Both authors thank DGICYT for research support under PB90-0258. Part of this work was done during a long-term visit by JL-G to the Department of Mathematical Analysis of La Laguna University during May-June, 1992. JL-G thanks his colleagues in La Laguna for their friendly hospitality.

\section{REFERENCES}

1. H. Amann, Fixed point equations and nonlinear eigenvalue problems in ordered Banach spaces, SIAM Rev. 18 (1976), 620-709.

2. J. Blat and K. J. Brown, Bifurcation of steady-state solutions in predator-prey and competition systems, Proc. Royal Soc. Edinburgh 97A (1984), 21-43.

3. $\mathrm{K}$. J. Brown and $\mathrm{Y}$. Du, Bifurcation and monotonicity in competition reaction-diffusion systems, Preprint.

4. R. Cantrell and C. Cosner, On the steady-state problem for the Lotka-Volterra competition model with diffusion, Houston J. Math. 13 (1987), 337-352.

5. __ Should a park be an island, SIAM J. Math. Appl. 53 (1993), 219-252.

6. C. Cosner and A. C. Lazer, Stable coexistence states in the Lotka Volterra competition model with diffusion, SIAM J. Appl. Math. 44 (1984), 1112-1132.

7. R. Courant and D. Hilbert, Methods of mathematical physics, Vol. II, Wiley Interscience, 1962.

8. M. G. Crandall and P. H. Rabinowitz, Bifurcation from simple eigenvalues, J. Funct. Anal. 8 (1971), 321-340.

9. $\ldots$, On the indices of fixed points of mappings in cones and applications, J. Math. Anal. Appl. 91 (1983), 131-151.

10. __ On positive solutions of some pairs of differential equations, Trans. Amer. Math. Soc. 284 (1984), 729-743.

11. $\ldots$ On the existence and uniqueness of positive solutions for competing species models with diffusion, Trans. Amer. Math. Soc. 326 (1991), 829-859.

12. J. C. Eilbeck, J. E. Furter, and J. López-Gómez, Coexistence in the competition model with diffusion, J. Differential Equations 107 (1994), 96-139.

13. A. Friedman, Partial differential equations of parabolic type, Prentice-Hall, Englewood Cliffs, N.J., 1964.

14. J. E. Furter and J. López-Gómez, On the existence and uniqueness of coexistence states for the Lotka-Volterra competition model with diffusion and spatial dependent coefficients, Nonlinear Anal., TMA (to appear).

15. D. Gilbarg and N. S. Trudinger, Elliptic partial differential equations of the second order, Springer-Verlag, Berlin, 1977.

16. J. K. Hale, L. T. Magalhães and, W. M. Oliva, An Introduction to infinite dimensional dynamical systems, geometric theory, Springer-Verlag, Berlin, 1984.

17. P. Hess, Periodic-parabolic boundary value problems and positivity, Pitman Res. Notes in Math., vol. 247, Pitman, 1991.

18. P. Hess and T. Kato, On some linear and nonlinear eigenvalue problems with an indefinite weight function, Comm. Partial Differential Equations 5 (1980), 999-1030.

19. T. Kato, Perturbation theory for linear operators, Springer-Verlag, Berlin, 1975.

20. A. Leung, Equilibria and stabilities for competing-species, reaction-diffusion equations with Dirichlet boundary data, J. Math. Anal. Appl. 73 (1980), 204-218.

21. A. Leung, Systems of nonlinear partial differential equations, Kluwer, Dordrecht-Boston, 1989.

22. J. López-Gómez, Positive periodic solutions of Lotka-Volterra R-D systems, Differential Integral Equations 5 (1992), 55-72. 
23. J. López-Gómez and M. Molina-Meyer, The maximum principle for cooperative weakly elliptic systems and some applications, Differential Integral Equations 7 (1994), 383-398.

24. J. López-Gómez and R. Pardo, Existence and uniqueness for some competition models with diffusion, C. R. Acad. Sci. Paris Ser. I 313 (1991), 933-938.

25. C. V. Pao, Coexistence and stability of a competition-diffusion system in population dynamics, J. Math. Anal. Appl. 83 (1981), 54-76.

26. C. V. Pao, Nonlinear parabolic and elliptic equations, Plenum Press, New York, 1992.

27. M. H. Protter and H. F. Weinberger, Maximum principles in differential equations, Prentice-Hall, Englewood Cliffs, N.J., 1967.

28. P. H. Rabinowitz, Some global results for nonlinear eigenvalue problems, J. Funct. Anal. 7 (1971), 487-513.

29. D. H. Sattinger, Topics in stability and bifurcation theory, Lecture Notes in Math., vol. 309, Springer-Verlag, 1973.

30. A. Schiaffino and A. Tesei, Competition systems with Dirichlet boundary conditions, J. Math. Biol. 15 (1982), 93-105.

Departamento de Matemática Aplicada, Universidad Complutense, 28040-Madrid, SPAIN

E-mail address: Julian@cara.ma.hw.ac.uk

Departamento de Análisis Matemático, Universidad de la laguna, 38271-La Laguna (TENERIFE), SPAIN

Current address: Department of Mathematics, Henot-Watt University, Riccarton Campus, Edinburgh EH14 4AS, U.K.

E-mail address: jsabina@ull.es 